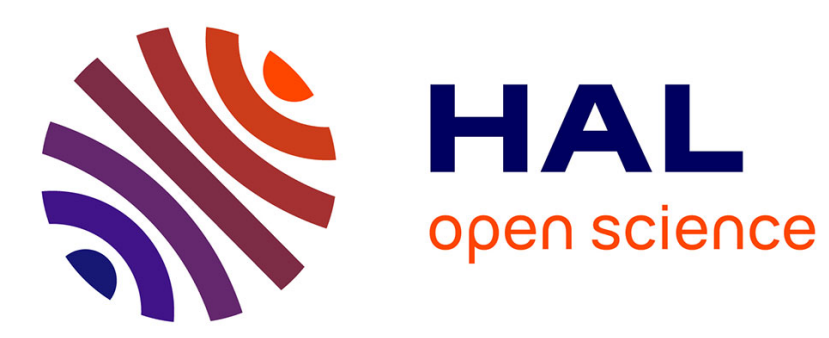

\title{
Infinite Horizon Optimal Control of Non-Convex Problems under State Constraints
}

Hélène Frankowska

\section{To cite this version:}

Hélène Frankowska. Infinite Horizon Optimal Control of Non-Convex Problems under State Constraints. Advances in Mathematical Economics, 23, Springer, 2020, Advances in Mathematical Economics, 978-981-15-0713-7. 10.1007/978-981-15-0713-7_2 . hal-02325736

\section{HAL Id: hal-02325736 \\ https://hal.science/hal-02325736}

Submitted on 24 Oct 2019

HAL is a multi-disciplinary open access archive for the deposit and dissemination of scientific research documents, whether they are published or not. The documents may come from teaching and research institutions in France or abroad, or from public or private research centers.
L'archive ouverte pluridisciplinaire HAL, est destinée au dépôt et à la diffusion de documents scientifiques de niveau recherche, publiés ou non, émanant des établissements d'enseignement et de recherche français ou étrangers, des laboratoires publics ou privés. 


\title{
INFINITE HORIZON OPTIMAL CONTROL OF NON-CONVEX PROBLEMS UNDER STATE CONSTRAINTS
}

\author{
HÉLÈNE FRANKOWSKA
}

\begin{abstract}
We consider the undiscounted infinite horizon optimal control problem under state constraints in the absence of convexity/concavity assumptions. Then the value function is, in general, nonsmooth. Using the tools of set-valued and nonsmooth analysis, the necessary optimality conditions and sensitivity relations are derived in such a framework. We also investigate relaxation theorems and uniqueness of solutions of the Hamilton-Jacobi-Bellman equation arising in this setting.
\end{abstract}

\section{INTRODUCTION}

The following infinite horizon optimal control problem is often present in models of mathematical economics and also in some engineering problems, (like, for instance, the general model of capital accumulation or design of asymptotically stabilizing controls),

$$
\operatorname{maximize} \int_{0}^{\infty} e^{-\lambda t} \ell(x(t), u(t)) d t
$$

over all trajectory-control pairs $(x, u)$ of the autonomous control system

$$
\left\{\begin{array}{l}
x^{\prime}(t)=f(x(t), u(t)), \quad u(t) \in U \quad \text { for a.e. } t \geq 0 \\
x(0)=x_{0}
\end{array}\right.
$$

subject to the state constraint $x(t)=\left(x_{1}(t), \ldots, x_{n}(t)\right) \geq 0$, where $e^{-\lambda t}$ is the discount factor for a given $\lambda>0$. Its history goes back to Ramsey [39]. Note that in the engineering problems maximization is often replaced by minimization, where results are similar after obvious adaptation of the involved data.

The literature addressing this problem deals with traditional questions of existence of optimal solutions, necessary and sufficient optimality conditions, sensitivity analysis, regularity of the value function, uniqueness of solutions of the associated Hamilton-Jacobi equation, ergodic theory, etc. The above problem is well investigated under the convexity/concavity assumptions implying concavity of the value function (also called the utility function). Then the powerful duality theory of convex analysis can be applied to get both necessary and sufficient optimality conditions and to show

This material is based upon work supported by the Air Force Office of Scientific Research under award number FA9550-18-1-0254. 
differentiability of the value function, see for instance $[13,42]$. Indeed, when data are convex/concave, necessary optimality conditions are also sufficient and the value function is differentiable whenever optimal trajectories are unique for every initial condition. This is, for instance, the case of strictly concave problems. In this way a clear picture of optimal solutions can be obtained.

In the general nonlinear case, however, typically the optimal solutions are not unique and necessary conditions are no longer sufficient for optimality. Even when state constraints are not involved, one can expect, at most, local Lipschitz continuity of the value function. Furthermore, if the discount factor is absent, the situation worsens, the value function being, in general, at most upper semicontinuous possibly taking infinite values. Thus, the classical tools can not be used any longer and have to be replaced by notions coming from the set-valued and non-smooth analysis. For instance, solutions of the Hamilton-Jacobi equation have to be understood in a generalized sense, e.g. viscosity solutions. Sensitivity relations become also more complex and involve generalized differentials, instead of derivatives.

Recently, while investigating necessary optimality conditions (in the absence of state constraints), a number of authors addressed more general setting of the infinite horizon problems, not involving the discount factor.

In the present paper, we consider the nonautonomous infinite horizon optimal control problem

$$
V\left(t_{0}, x_{0}\right)=\sup \int_{t_{0}}^{\infty} L(t, x(t), u(t)) d t
$$

over all trajectory-control pairs $(x, u)$ of the control system

$$
\left\{\begin{array}{l}
x^{\prime}(t)=f(t, x(t), u(t)), \quad u(t) \in U(t) \quad \text { for a.e. } t \geq t_{0} \\
x\left(t_{0}\right)=x_{0},
\end{array}\right.
$$

satisfying the state constraint

$$
x(t) \in K \text { for all } t \geq t_{0},
$$

where $L: \mathbb{R}_{+} \times \mathbb{R}^{n} \times \mathbb{R}^{m} \rightarrow \mathbb{R}, f: \mathbb{R}_{+} \times \mathbb{R}^{n} \times \mathbb{R}^{m} \rightarrow \mathbb{R}^{n}$ are given mappings, $\mathbb{R}_{+}=[0,+\infty), t_{0} \in \mathbb{R}_{+}, x_{0} \in \mathbb{R}^{n}, U: \mathbb{R}_{+} \leadsto \mathbb{R}^{m}$ is a measurable set-valued map with closed nonempty images and $K \subset \mathbb{R}^{n}$ is nonempty and closed. Selections $u(t) \in U(t)$ are supposed to be Lebesgue measurable and are called controls. The above setting subsumes the classical infinite horizon optimal control problem when $f$ and $U$ are time independent, $L(t, x, u)=e^{-\lambda t} \ell(x, u)$ for some mapping $\ell: \mathbb{R}^{n} \times \mathbb{R}^{m} \rightarrow \mathbb{R}_{+}$and $\lambda>0, t_{0}=0$.

Infinite horizon problems exhibit many phenomena not arising in the context of finite horizon problems and their study is still going on, even in the absence of state constraints, see $[1,2,3,4,5,6,33,36,37,38,40,43,44]$ and their bibliographies. Among such phenomena let us recall that already in 70ies Halkin, see [32] and also [36], observed that in the necessary optimality conditions for an infinite horizon problem it may happen that the co-state of the maximum principle is different from zero at infinity and that only 
abnormal maximum principles hold true (even for problems without state constraints). Such phenomena do not occur for the finite horizon problems.

The presence of state-constraints drastically changes the maximum principle: as in the case of finite horizon problems, a trajectory-control pair satisfying simultaneously the unconstrained Pontryagin maximum principle and the state constraint may be unique and not necessarily optimal. As a consequence, one has to work with discontinuous co-states and more complex adjoint systems.

While the existence theories for problems with or without state constraints are essentially the same (on the domain $\operatorname{dom}(V)$ of the value function), this is no longer the case in what concerns optimality conditions and sensitivity relations. Since 70ies many paths were exploited in the literature to derive necessary optimality conditions for the infinite horizon problem when $K=\mathbb{R}^{n}$. The most immediate one consists in replacing the infinite horizon problem by a family of (finite horizon) Bolza problems on intervals $\left[t_{0}, T\right]$ for $T>t_{0}$ (that is substituting $\infty$ by $T$ in the definition of the cost (1)) and using the known results for the Bolza problem. In particular, the first order necessary condition for each Bolza problem takes the form of the maximum principle: if $(\bar{x}, \bar{u})$ is an optimal trajectory-control pair for the Bolza problem at the initial condition $\left(t_{0}, x_{0}\right)$, then the solution $p_{T}:=p$ of the adjoint system

$$
-p^{\prime}(t)=f_{x}(t, \bar{x}(t), \bar{u}(t))^{*} p(t)+L_{x}(t, \bar{x}(t), \bar{u}(t)), \quad p(T)=0
$$

satisfies the maximality condition

$$
\langle p(t), f(t, \bar{x}(t), \bar{u}(t))\rangle+L(t, \bar{x}(t), \bar{u}(t))=H(t, \bar{x}(t), p(t)) \text { a.e. in }\left[t_{0}, T\right],
$$

where the Hamiltonian $H: \mathbb{R}_{+} \times \mathbb{R}^{n} \times \mathbb{R}^{n} \rightarrow \mathbb{R}$ is defined by

$$
H(t, x, p):=\sup _{u \in U(t)}(\langle p, f(t, x, u)\rangle+L(t, x, u)) .
$$

We underline that the transversality condition $p(T)=0$ is due to the fact that there is no additional cost term depending on the final state $x(T)$ in the considered Bolza problems.

Recall that if $H(t, \cdot, \cdot)$ is differentiable, then the adjoint system and the maximality condition can be equivalently written in the form of the Hamiltonian system: for a.e. $t \in\left[t_{0}, T\right]$

$$
\left\{\begin{aligned}
-p^{\prime}(t) & =H_{x}(t, \bar{x}(t), p(t)), & & p(T)=0 \\
\bar{x}^{\prime}(t) & =H_{p}(t, \bar{x}(t), p(t)), & & \bar{x}\left(t_{0}\right)=x_{0} .
\end{aligned}\right.
$$

In general, however, $H(t, \cdot, \cdot)$ is not differentiable and one writes instead a Hamiltonian differential inclusion involving generalized gradients of $H(t, \cdot, \cdot)$, see $[19,45]$.

Then, taking limits of co-states $p_{T}(\cdot)$ when $T \rightarrow \infty$ is expected to lead to the maximum principle of the infinite horizon problem. This approach requests however some important modifications, due to the fact that the restrictions of an optimal solution $(\bar{x}, \bar{u})$ of the infinite horizon problem to the 
finite intervals $\left[t_{0}, T\right]$ may be not optimal for the Bolza problems. To overcome this difficulty, some authors add the end point constraint $x(T)=\bar{x}(T)$. With such an additional constraint the restriction of $(\bar{x}, \bar{u})$ to the time interval $\left[t_{0}, T\right]$ becomes optimal for the above Bolza problem. This leads, however, to possibly abnormal maximum principles for finite horizon problems, and, in fine, admits necessary optimality conditions not involving the cost function $L$. Also the transversality condition at time $T$ does disappear, becoming $-p(T) \in N_{\{\bar{x}(T)\}}(\bar{x}(T))=\mathbb{R}^{n}$ (normal cone to the singleton $\{\bar{x}(T)\}$ at $\bar{x}(T))$.

Another way to deal with this issue is to modify the very definition of optimal solution, cf. $[3,4,17,32,46]$. However, the notions like overtaking (or weakly overtaking) optimal controls do not have appropriate existence theory. More precisely, no specific sufficient conditions were proposed to guarantee the existence of overtaking optimal controls. This is the reason why we prefer to stick to the classical notion of optimality, where sufficient condition for the existence of optimal controls are well understood.

An alternative approach to the discounted infinite horizon problems consists in modification of the cost function in such a way that restrictions of $(\bar{x}, \bar{u})$ to intervals $\left[t_{0}, T\right]$ are locally optimal for the Bolza problems, cf. $[35,40,47]$. The presence of the discount factor allows then to pass to the limit of the (finite horizon) maximum principles and to conclude that a costate satisfies the adjoint system, the maximality condition and vanishes at infinity. We would like to underline here that such a "terminal" transversality condition at infinity is a consequence of the assumptions on data. This differs substantially from the finite horizon settings, where the transversality condition at the terminal time is an independent requirement. This approach exploits the value function $V$. Actually, in [35] $V$ is supposed to be $C^{1}$ (which is a too strong request) to get these conclusions, while in [47] it is Lipschitz continuous. Furthermore, for the discounted problems considered in $[7,35,40,47]$ the sensitivity relations helped to write a transversality condition also at the initial time.

Another way to derive the maximum principle (still when there are no state constraints) relies on the duality theory on weighted Sobolev spaces with respect to the measure $e^{-\lambda t} d t$ (or more general measures), cf. [7, 36, $37,44]$.

In the absence of the discount factor, the question of necessary conditions is quite challenging, because, unlike for classical finite horizon problems, transversality conditions are not immediate. We refer to [2] for an extended overview of the literature devoted to transversality conditions and for bibliographical comments and also to [3] for a further discussion.

The major difficulties in dealing with state constrained problems are due to the fact that for a given optimal solution $(\bar{x}, \bar{u})$ of $(1)$ - (3), small perturbations of the initial state $\left(t_{0}, x_{0}\right)$ or of the control $\bar{u}$ may result in trajectories violating state constraints. This creates obstacles for the direct application 
of classical variational methods (as for instance needle perturbations) to derive necessary optimality conditions. In addition, it may happen that the value function $V$ takes infinite values and is discontinuous. For this reason the classical tools of optimal control theory like Hamilton-Jacobi equation and its viscosity solutions are no longer adapted.

Recall that (continuous) viscosity solutions to first-order partial differential equations were introduced in [20, 21] by Crandall, Evans, and Lions to investigate Hamilton-Jacobi equations not admitting classical solutions. In particular, given $T>0$ and a continuous "terminal" function $g_{T}(\cdot)$, they proved existence and uniqueness of continuous solutions to

$$
-\partial_{t} V+H\left(t, x,-\nabla_{x} V\right)=0 \quad \text { on }(0, T) \times \mathbb{R}^{n}, \quad V(T, \cdot)=g_{T}(\cdot),
$$

when the Hamiltonian $H$ is continuous. In the absence of state constraints, under mild assumptions, the value function of the Bolza problem is the unique viscosity solution of (4) provided it is bounded and uniformly continuous. Some sufficient conditions (in the form of an inward pointing assumption) for continuity of the value function for a discounted state constrained infinite horizon problem can be found in [41], when $f, \ell$ are time independent and $K$ is a compact set having smooth boundary. It is shown in [41] that the value function is the unique viscosity solution to a corresponding stationary Hamilton-Jacobi equation. However such a framework leaves aside the conical state constraints and the time dependent case, because, as it was shown later on, arguments of [41] no longer apply in the non-autonomous case whenever the time dependence is merely continuous. Some extensions, when $K$ is a locally compact set with possibly nonsmooth boundary, can be found in [29].

In the class of finite horizon state-constrained problems, the Mayer one has been successfully investigated by many authors, see for instance $[12,15$, 19, 26, 31, 45] and their bibliographies. Also, it is well known that the Bolza problem can be stated, in an equivalent way, as the Mayer one (without loosing optimality of solutions). This created a favorable background to approach the infinite horizon problems under state constraints.

In [14], in the absence of state constraints, we proposed to use systematically the dynamic programming and to add to the integral functional of the Bolza problem defined on $[0, T]$ the discontinuous (in general) cost function $V(T, \cdot)$. That is for $g_{T}(\cdot):=V(T, \cdot)$ we considered the Bolza problem

$$
\operatorname{maximize}\left(g_{T}(x(T))+\int_{t_{0}}^{T} L(t, x(t), u(t)) d t\right)
$$

over all trajectory-control pairs $(x, u)$ of $(2)$ defined on $\left[t_{0}, T\right]$.

The above finite horizon Bolza problem enjoys the following crucial property: restrictions of an optimal solution $(\bar{x}, \bar{u})$ of the infinite horizon problem to the finite intervals $\left[t_{0}, T\right]$ with $T>t_{0}$ are optimal for the Bolza problems. Also, for every $T>t_{0}$ the value function of the Bolza problem coincides with $V$ on $\left[t_{0}, T\right] \times \mathbb{R}^{n}$. 
Let us underline however that this new problem involves the, possibly discontinuous, cost function $V(T, \cdot)$ and for this reason one needs nonsmooth maximum principles derived for finite horizon problems to express necessary optimality conditions.

Such an approach allowed us, by passing to the limit when $T \rightarrow \infty$, to get the maximum principle and sensitivity relations and has lead to the transversality condition at the initial time. In particular, this result contains the maximum principle (the sensitivity relation and the initial time transversality condition providing an additional information). Furthermore, we have shown the validity of the relaxation theorems whenever the value function of the relaxed problem is continuous with respect to the state variable. It could be interesting to extend also the second order sensitivity relations from [16] to the case of infinite horizon problems.

The present paper discusses results of the same nature but in the presence of state constraints. We start by proving the upper semicontinuity of the value function under the classical assumptions guaranteeing existence of optimal solutions. These assumptions involve convexity of sets

$$
F(t, x):=\{(f(t, x, u), L(t, x, u)-r): u \in U(t) \text { and } r \geq 0\} .
$$

We would like to emphasize that this condition (of Cesari-Olech type) is classical in the existence theory of optimal control and does not yield concavity of the value function. To investigate uniqueness of solutions to the Hamilton-Jacobi equation, we also need a sufficient condition for $V$ to vanish at infinity. For this aim we shall impose the following assumption

$\left(H_{0}\right)$ : There exists $S>0$ such that $|L(t, x, u)| \leq \alpha(t)$ for a.e. $t \geq S$ and all $x \in K, u \in U(t)$, where $\alpha:[S,+\infty) \rightarrow \mathbb{R}_{+}$is integrable on $[S,+\infty)$ (see Section 4 for more details.)

Under this assumption

$$
\lim _{t \rightarrow \infty} \sup _{x \in \operatorname{dom}(V(t, \cdot))}|V(t, x)|=0 .
$$

This "terminal" condition at infinity replaces the final condition of (4).

When sets $F(t, x)$ are not convex, then it is usual to consider the so-called relaxed problems and speak about generalized solutions. They can be stated either by using the probability measures and relaxed controls or, equivalently, by considering the convexified infinite horizon problem

$$
V^{r e l}\left(t_{0}, x_{0}\right)=\sup \int_{t_{0}}^{\infty}\left(\sum_{i=0}^{n} \lambda_{i}(t) L\left(t, x(t), u_{i}(t)\right)\right) d t
$$

over all trajectory-control pairs of the relaxed constrained control system

$$
\left\{\begin{array}{l}
x^{\prime}(t)=\sum_{i=0}^{n} \lambda_{i}(t) f\left(t, x(t), u_{i}(t)\right), u_{i}(t) \in U(t), \lambda_{i}(t) \geq 0, \sum_{i=0}^{n} \lambda_{i}(t)=1 \\
x\left(t_{0}\right)=x_{0}, x(t) \in K \forall t \geq t_{0},
\end{array}\right.
$$

where $u_{i}(\cdot), \lambda_{i}(\cdot)$ are Lebesgue measurable on $\mathbb{R}_{+}$for $i=0, \ldots, n$. Clearly $V^{r e l} \geq V$. Furthermore, for the relaxed problem the corresponding sets $F(t, x)$ are convex. When $K=\mathbb{R}^{n}$, assumption $\left(H_{0}\right)$ allows to prove a relaxation 
theorem whenever the sets $\{(f(t, x, u), L(t, x, u)): u \in U(t)\}$ are compact and to identify a more complex relaxed problem when they are neither closed nor bounded, see Section 5 below for more details. In this way we extend the relaxation theorem from [14] to the case of unbounded sets $\{(f(t, x, u), L(t, x, u)): u \in U(t)\}$.

For the finite horizon problems, one can find in the literature some relaxation theorems concerned with a single relaxed solution $x(\cdot)$ of a differential inclusion whose right-hand side has compact integrably bounded values on a tubular neighborhood of $x(\cdot)$, cf. $[19,45]$. In our finite horizon relaxation Theorem 5.3 below we show that for control systems such assumptions can be skipped. Even though Theorem 5.3 concerns all the relaxed trajectories, its proof can be localized to a tubular neighborhood of a fixed relaxed trajectory. Then assumptions can be localized as well without requiring compactness and integral boundedness imposed in $[19,45]$. In this respect, Theorem 5.3 would imply a new result also in the setting of $[19,45]$.

The situation changes drastically, even in the finite horizon framework, when state constraints are present. For instance the original control system may not have any feasible trajectories, while the relaxed system does. Then, in order to get relaxation theorems, one needs the so called relaxed inward pointing condition (IPC ${ }^{\text {rel }}$ ) linking $f$ with tangents to $K$ at the boundary points of $K$, that we recall in Section 6 . This condition expresses the compatibility of dynamics with state constraints. It was introduced in [26] to derive the normal maximum principle and the sensitivity relations for a state-constrained Mayer problem with locally Lipschitz cost function.

In Section 8 we show that it can be exploited as well to get similar results for the state constrained infinite horizon problem, provided $V(t, \cdot)$ is locally Lipschitz on $K$ for all large $t$. (IPC $\left.{ }^{r e l}\right)$ is an alternative to the inward pointing condition from [41] to prove the so called Neighboring Feasible Trajectory theorem, when dynamics depend on time. The inward pointing condition is much simpler than $\left(I P C^{r e l}\right)$, but, unfortunately, is not convenient to work with data depending measurably (or even continuously) on time and state constraints having nonsmooth boundaries. It is not difficult to realize that under assumptions imposed in [41], the inward pointing condition is equivalent to $\left(I P C^{r e l}\right)$.

Recall that for problems without state constraints the sensitivity relation $p(t)=V_{x}(t, \bar{x}(t))$ has a significant economic interpretation (see for instance [1], [42]): the co-state $p$ (of the maximum principle) is the shadow price describing the contribution to the value function of a unit increase of capital $x$.

When the value function is merely locally Lipschitz on $\mathbb{R}_{+} \times K$, the sensitivity relation is more complex and takes the form

$$
(-H(t, \bar{x}(t), q(t)), q(t)) \in \partial V(t, \bar{x}(t)),
$$

where $q$ is the adjoint state (of bounded variation) and $\partial V$ denotes the generalized gradient of $V$ (defined in Section 8 taking into consideration the state 
constraints). In Section 8 we derive the maximum principle augmented by the above sensitivity relation for a state constrained infinite horizon problem. An important future of the obtained here necessary optimality condition is its normality.

Local Lipschitz continuity of $V$ for infinite horizon problems under state constraints was recently investigated in [10]. On the other hand, uniqueness of upper semicontinuous solutions of the associated Hamilton-Jacobi equation was studied in [11]. In Section 9 we show uniqueness of locally Lipschitz solutions of the Hamilton-Jacobi equation by arguments simpler than those in $[11]$.

The outline of the paper is as follows. In Section 2, we recall some definitions from set-valued and nonsmooth analysis. In Sections 3, we introduce the value function $V$ and basic assumptions that imply in Section 4 the upper semicontinuity of $V$. In Sections 5 and 6 we discuss the relaxation results for problems without and with state constraints, respectively, and in Section 7 we describe the link between the finite and infinite horizon problems. Section 8 is devoted to the maximum principle and sensitivity relations, while Section 9 deals with the uniqueness of solutions to the Hamilton-Jacobi equation.

\section{Preliminaries and notations}

Denote by $L_{\text {loc }}^{1}\left(\mathbb{R}_{+} ; \mathbb{R}_{+}\right)$the set of all locally integrable function $\psi: \mathbb{R}_{+} \rightarrow$ $\mathbb{R}_{+}$. For any $\psi \in L_{\text {loc }}^{1}\left(\mathbb{R}_{+} ; \mathbb{R}_{+}\right)$and $\sigma>0$ define

$$
\theta_{\psi}(\sigma)=\sup \left\{\int_{J} \psi(\tau) d \tau: J \subset \mathbb{R}_{+}, \mathcal{M}(J) \leqslant \sigma\right\},
$$

where $\mathcal{M}(J)$ stands for the Lebesgue measure of $J$. Denote by $\mathcal{L}_{\text {loc }}$ the subset of all $\psi \in L_{\text {loc }}^{1}\left(\mathbb{R}_{+} ; \mathbb{R}_{+}\right)$such that $\lim _{\sigma \rightarrow 0} \theta_{\psi}(\sigma)=0$. Notation $W_{\text {loc }}^{1,1}\left(\mathbb{R}_{+} ; \mathbb{R}^{n}\right)$ stands for the set of locally absolutely continuous functions on $\mathbb{R}_{+}$. For $\psi: \mathbb{R}_{+} \rightarrow \mathbb{R}$ and $t_{0} \geq 0$ define

$$
\int_{t_{0}}^{\infty} \psi(t) d t=\lim _{T \rightarrow+\infty} \int_{t_{0}}^{T} \psi(t) d t
$$

provided the above limit does exist.

Let $X$ be a normed space, $B(x, R)$ be the closed ball in $X$ centered at $x \in X$ with radius $R>0$ and set $B:=B(0,1)$. For a nonempty subset $C \subset X$ we denote its interior by $\operatorname{int}(C)$, its boundary by bd $(C)$, its convex hull by co $C$, its closed convex hull by $\overline{\mathrm{co}} C$, and the distance from $x \in X$ to $C$ by $d_{C}(x):=\inf \left\{|x-y|_{X}: y \in C\right\}$. If $X=\mathbb{R}^{n}$, the negative polar cone to $C$ is given by $C^{-}=\left\{p \in \mathbb{R}^{n}:\langle p, c\rangle \leqslant 0 \quad \forall c \in C\right\}$, where $\langle\cdot, \cdot\rangle$ is the scalar product in $\mathbb{R}^{n}$. The unit sphere in $\mathbb{R}^{n}$ is denoted by $S^{n-1}$.

Let $\mathcal{T} \subset \mathbb{R}^{k}$ be nonempty and $\left\{A_{\tau}\right\}_{\tau \in \mathcal{T}}$ be a family of subsets of $\mathbb{R}^{n}$. The upper and lower limits, in the Péano-Kuratowski sense, of $A_{\tau}$ at $\tau_{0} \in \mathcal{T}$ are 
the closed sets defined respectively by

$$
\begin{aligned}
& \operatorname{Limsup}_{\tau \rightarrow \mathcal{T}_{0}} A_{\tau}=\left\{v \in \mathbb{R}^{n}: \liminf _{\tau \rightarrow \mathcal{T} \tau_{0}} d_{A_{\tau}}(v)=0\right\}, \\
& \operatorname{Liminf}_{\tau \rightarrow \mathcal{T} \tau_{0}} A_{\tau}=\left\{v \in \mathbb{R}^{n}: \limsup _{\tau \rightarrow \mathcal{T} \tau_{0}} d_{A_{\tau}}(v)=0\right\},
\end{aligned}
$$

where $\rightarrow \mathcal{T}$ stands for the convergence in $\mathcal{T}$ and $d_{A_{\tau}}(v)=+\infty$ whenever $A_{\tau}=\emptyset$. See for instance [8] for properties of these set limits.

Let $K \subset \mathbb{R}^{n}$ and $x \in K$. The contingent cone to $K$ at $x$ consists of all $v \in \mathbb{R}^{n}$ such that for some sequences $h_{i} \rightarrow 0+, v_{i} \rightarrow v$ we have $x+h_{i} v_{i} \in K$. The limiting normal cone to a closed subset $K \subset \mathbb{R}^{n}$ at $x \in K$ is given by

$$
N_{K}^{L}(x):=\operatorname{Limsup}_{y \rightarrow K} T_{K}(y)^{-} .
$$

It is well known that if $x$ lies on the boundary of $K$, then $N_{K}^{L}(x)$ is not reduced to zero. The Clarke tangent and normal cones to $K$ at $x$ are defined by $C_{K}(x)=\left(N_{K}^{L}(x)\right)^{-}$and $N_{K}(x)=C_{K}(x)^{-}$, respectively. Note that $N_{K}(x)=\overline{\operatorname{co}} N_{K}^{L}(x)$ and set $N_{K}^{1}(x):=N_{K}(x) \cap S^{n-1}$.

For $\varphi: \mathbb{R}^{n} \rightarrow \mathbb{R} \cup\{ \pm \infty\}$ denote by $\operatorname{dom}(\varphi)$ the domain of $\varphi$, that is the set of all $x \in \mathbb{R}^{n}$ such that $\varphi(x)$ is finite and by $\operatorname{epi}(\varphi)$ and $h y p(\varphi)$, respectively, its epigraph and hypograph. For any $x \in \operatorname{dom}(\varphi)$ the upper and lower (contingent) directional derivatives of $\varphi$ at $x$ in the direction $y \in \mathbb{R}^{n}$ are defined respectively by

$$
\begin{aligned}
& D_{\downarrow} \varphi(x) y=\limsup _{z \rightarrow y, h \rightarrow 0+} \frac{\varphi(x+h z)-\varphi(x)}{h}, \\
& D_{\uparrow \varphi}(x) y=\liminf _{z \rightarrow y, h \rightarrow 0+} \frac{\varphi(x+h z)-\varphi(x)}{h}
\end{aligned}
$$

and the Fréchet superdifferential $\partial^{+} \varphi(x)$ (resp. subdifferential $\partial^{-} \varphi(x)$ ) of $\varphi$ at $x$ by

$$
p \in \partial^{+} \varphi(x) \Longleftrightarrow \limsup _{y \rightarrow x} \frac{\varphi(y)-\varphi(x)-\langle p, y-x\rangle}{|y-x|} \leq 0
$$

and

$$
p \in \partial^{-} \varphi(x) \Longleftrightarrow \liminf _{y \rightarrow x} \frac{\varphi(y)-\varphi(x)-\langle p, y-x\rangle}{|y-x|} \geq 0 .
$$

By [22], $p \in \partial^{+} \varphi(x)$ if and only if $(-p,+1) \in T_{h y p(\varphi)}(x, \varphi(x))^{-}$and $p \in$ $\partial^{-} \varphi(x)$ if and only if $(p,-1) \in T_{e p i(\varphi)}(x, \varphi(x))^{-}$. Furthermore,

$$
\begin{aligned}
& \partial^{+} \varphi(x)=\left\{p:\langle p, y\rangle \geq D_{\downarrow} \varphi(x) y \forall y \in \mathbb{R}^{n}\right\}, \\
& \partial^{-} \varphi(x)=\left\{p:\langle p, y\rangle \leq D_{\uparrow} \varphi(x) y \forall y \in \mathbb{R}^{n}\right\} .
\end{aligned}
$$

To compare with sub and superdifferentials used in the theory of viscosity solutions, let us emphasize that the very same arguments as those of [21, Proof of Proposition 1.1] imply the following result.

Proposition 2.1. Let $\varphi: \mathbb{R}^{n} \rightarrow \mathbb{R} \cup\{-\infty\}$ be Lebesgue measurable. For any $x \in \operatorname{dom}(\varphi)$, a vector $p \in \partial^{+} \varphi(x)$ if and only if there exists a continuous mapping $\psi: \mathbb{R}^{n} \rightarrow \mathbb{R}$ such that $\psi(x)=\varphi(x), \psi(y)>\varphi(y)$ for all $y \neq x$ and the Fréchet derivative of $\psi$ at $x$ exists and is equal to $p$. 
Actually, in [21] $\varphi$ is continuous and $\psi \in C^{1}$. However for discontinuous mappings, in general, $\psi$ constructed in $[21]$ is not continuously differentiable. A similar result can be also stated for the subdifferential $\partial^{-} \varphi(x)$.

Clearly, for all $p \in \mathbb{R}^{n}$ and $q \in \mathbb{R}$ satisfying $(p, q) \in N_{e p i(\varphi)}^{L}(x, \varphi(x))$ we have $q \leq 0$. Furthermore, if $q<0$, then $(p, q) \in N_{e p i(\varphi)}^{L}(x, \varphi(x))$ if and only if $(p /|q|,-1) \in N_{e p i(\varphi)}^{L}(x, \varphi(x))$. Any $p \in \mathbb{R}^{n}$ satisfying $(p,-1) \in N_{e p i(\varphi)}^{L}(x, \varphi(x))$ is called a limiting subgradient of $\varphi$ at $x$. The set of all limiting subgradients of $\varphi$ at $x$ is denoted by $\partial^{L,-} \varphi(x)$.

Consider $\varphi: \mathbb{R}^{n} \rightarrow \mathbb{R}$, Lipschitz around a given $x \in \mathbb{R}^{n}$, and denote by $\nabla \varphi(\cdot)$ its gradient, which, by the Rademacher theorem, exists a.e. in a neighborhood of $x$. The Clarke generalized gradient of $\varphi(\cdot)$ at $x$ is defined by

$$
\partial \varphi(x):=\operatorname{coLimsup}_{y \rightarrow x}\{\nabla \varphi(y)\} .
$$

It is well known that $\partial \varphi(x)=c o \partial^{L,-} \varphi(x)$, see [19].

\section{VALUE FUNCTION OF THE INFINITE HORIZON PROBLEM}

Consider the non-autonomous infinite horizon optimal control problem (1), (2), (3) with data as described in the introduction. In particular, $U(\cdot)$ is Lebesgue measurable and has closed nonempty images. Every Lebesgue measurable $u: \mathbb{R}_{+} \rightarrow \mathbb{R}^{m}$ satisfying $u(t) \in U(t)$ a.e. is called a control and the set of all controls is denoted by $\mathcal{U}$. Note that to state (2) we need controls to be defined only on $\left[t_{0},+\infty\right)$. However, since throughout the paper the time interval varies, to avoid additional notations and without any loss of generality, we consider controls defined on $[0,+\infty)$. We underline that, by the measurable selection theorem, for any measurable selection $u(t) \in U(t)$ for $t \in\left[t_{0}, \infty\right)$ we can find $\tilde{u} \in \mathcal{U}$ such that $\tilde{u}=u$ on $\left[t_{0}, \infty\right)$.

\section{Assumptions (H1):}

i) There exists $c \in L_{\text {loc }}^{1}\left(\mathbb{R}_{+} ; \mathbb{R}_{+}\right)$such that for a.e. $t \geq 0$,

$2\langle f(t, x, u), x\rangle \leq c(t)\left(1+|x|^{2}\right),|f(t, 0, u)| \leq c(t) \forall x \in \mathbb{R}^{n}, u \in U(t) ;$

ii) For every $R>0$, there exist $c_{R} \in L_{\text {loc }}^{1}\left(\mathbb{R}_{+} ; \mathbb{R}_{+}\right)$and a modulus of continuity $\omega_{R}: \mathbb{R}_{+} \times \mathbb{R}_{+} \rightarrow \mathbb{R}_{+}$such that for a.e. $t \in \mathbb{R}_{+}, \omega_{R}(t, \cdot)$ is increasing, $\lim _{r \rightarrow 0+} \omega_{R}(t, r)=0$ and for every $u \in U(t)$ and $x, y \in$ $B(0, R)$,

$|f(t, x, u)-f(t, y, u)| \leq c_{R}(t)|x-y|,|L(t, x, u)-L(t, y, u)| \leq \omega_{R}(t,|x-y|) ;$

iii) The mappings $f, L$ are Carathéodory, that is measurable in $t$ and continuous in $x, u$;

iv) There exists $\beta \in L_{\text {loc }}^{1}\left(\mathbb{R}_{+} ; \mathbb{R}_{+}\right)$and an increasing function $\phi: \mathbb{R}_{+} \rightarrow$ $\mathbb{R}_{+}$such that for a.e. $t \in \mathbb{R}_{+}$,

$$
|L(t, x, u)| \leq \beta(t) \phi(|x|), \quad \forall x \in K, u \in U(t) ;
$$

v) There exists $S>0$ such that $L(t, x, u) \leq \alpha(t)$ for a.e. $t \geq S$ and all $x \in K, u \in U(t)$, where $\alpha:[S,+\infty) \rightarrow \mathbb{R}_{+}$is integrable on $[S,+\infty)$. 
vi) For a.e. $t \in \mathbb{R}_{+}$and for all $x \in \mathbb{R}^{n}$ the set

$$
F(t, x):=\{(f(t, x, u), L(t, x, u)-r): u \in U(t) \text { and } r \geq 0\}
$$

is closed and convex.

\section{Remark 3.1.}

a) In some results below (H1) i) or (vi) will be skipped.

b) Assumptions (H1) i), ii) and iii) imply that for every $\left(t_{0}, x_{0}\right) \in \mathbb{R}_{+} \times \mathbb{R}^{n}$ and $u \in \mathcal{U}$ there exists a unique solution defined on $\left[t_{0}, \infty\right)$ of the system

$$
x^{\prime}=f(t, x, u(t))
$$

satisfying $x\left(t_{0}\right)=x_{0}$.

c) Note that assumption (H1) i) holds true whenever

(6) $\exists \theta \in L_{\text {loc }}^{1}\left(\mathbb{R}_{+} ; \mathbb{R}_{+}\right),|f(t, x, u)| \leq \theta(t)(|x|+1), \forall t \in \mathbb{R}_{+}, x \in \mathbb{R}^{n}, u \in U(t)$.

d) Note that iv) and v) imply that for every feasible trajectory control pair of (5) defined on $\left[t_{0}, \infty\right)$ the $\operatorname{limit}_{\lim } \rightarrow \infty \int_{t_{0}}^{T} L(t, x(t), u(t)) d t$ does exist and belongs to $[-\infty, \infty)$.

e) Even though it may seem, at first glance, that conditions like i), ii), iv), vi) yield compactness of sets $U(t)$, since $f(t, x, \cdot)$ is genuinely nonlinear and merely continuous, (H1) does not imply boundedness of sets $U(t)$.

Consider $0 \leq a \leq b$ and $u \in \mathcal{U}$. An absolutely continuous function $x$ : $[a, b] \rightarrow \mathbb{R}^{n}$ satisfying $x^{\prime}(t)=f(t, x(t), u(t))$ a.e. in $[a, b]$ is called a solution of (5) corresponding to the control $u(\cdot)$ and $(x, u)$ is called a trajectorycontrol pair of (5) on $[a, b]$. If moreover $x(t) \in K$ for all $t \in[a, b]$, then such a solution $x$ is called feasible and $(x, u)$ is called a feasible trajectory-control pair on $[a, b]$. It may happen that for some control $u(\cdot)$ and $\left(t_{0}, x_{0}\right) \in \mathbb{R}_{+} \times K$ the system (5) does not have any feasible solution satisfying $x\left(t_{0}\right)=x_{0}$. If $(x, u):[a, \infty) \rightarrow \mathbb{R}^{n} \times \mathbb{R}^{m}$ is so that for every $b>a$, the restriction of $(x, u)$ to $[a, b]$ is a trajectory-control pair of $(5)$ on $[a, b]$, then $(x, u)$ is called a trajectory-control pair on $[a, \infty)$. It is feasible, if $x(t) \in K$ for every $t \geq a$.

The function $V: \mathbb{R}_{+} \times K \rightarrow \mathbb{R}_{+}$defined by (1), (2), (3) is called the value function of the infinite horizon problem. If for a given $\left(t_{0}, x_{0}\right) \in \mathbb{R}_{+} \times K$ no trajectory of $(2),(3)$ does exist, we set $V\left(t_{0}, x_{0}\right)=-\infty$. This choice is dictated by the fact that, in general, $V$ is discontinuous and, under the (classical) assumptions (H1), it is upper semicontinuous on $\operatorname{dom}(V) \subset \mathbb{R}_{+} \times$ $K$, see the next section. To preserve its upper semicontinuity on $\mathbb{R}_{+} \times \mathbb{R}^{n}$ we set $V=-\infty$ on $\left(\mathbb{R}_{+} \times \mathbb{R}^{n}\right) \backslash \operatorname{dom}(V)$. Clearly, under assumption (H1) iv), if for some $T \geq 0$ the set $\operatorname{dom}(V(T, \cdot)) \neq \emptyset$, then $\operatorname{dom}(V(t, \cdot)) \neq \emptyset$ for every $t \geq T$.

Denote by $x\left(\cdot ; t_{0}, x_{0}, u\right)$ the trajectory of $(5)$ corresponding to the control $u$ and satisfying $x\left(t_{0}\right)=x_{0}$. By Gronwall's lemma and (H1) i), for all $\left(t_{0}, x_{0}\right) \in$ $\mathbb{R}_{+} \times \mathbb{R}^{n}$,

$$
\left|x\left(t ; t_{0}, x_{0}, u\right)\right|^{2} \leq\left(\left|x_{0}\right|^{2}+\int_{t_{0}}^{t} c(s) d s\right) e^{\int_{t_{0}}^{t} c(s) d s} \quad \forall t \geq t_{0} .
$$


Moreover, setting

$$
M_{t}(T, r)^{2}=\left(r^{2}+\int_{t}^{T} c(s) d s\right) e^{\int_{t}^{T} c(s) d s} \quad \forall T \geq t \geq 0, r \geq 0,
$$

the following holds true: for all $r \geq 0$ and $u \in \mathcal{U}$

$$
\left|x_{0}\right| \leq r \Longrightarrow\left|x\left(t ; t_{0}, x_{0}, u\right)\right| \leq M_{t_{0}}(t, r) \quad \forall t \geq t_{0} .
$$

The above bound, together with the assumption (H1) ii) and the Gronwall lemma, yield the local Lipschitz dependence of trajectories on the initial conditions: for all $r, T>0$, for every $t_{0} \in[0, T]$ and $x_{0}, x_{1} \in B(0, r)$,

$$
\left|x\left(t ; t_{0}, x_{1}, u\right)-x\left(t ; t_{0}, x_{0}, u\right)\right| \leq\left|x_{1}-x_{0}\right| e^{\int_{t_{0}}^{t} c_{M_{t_{0}}(T, r)}(s) d s} \forall t \in\left[t_{0}, T\right] .
$$

Given a feasible trajectory-control pair $(x, u)$, define

$$
\int_{t_{0}}^{\infty} L(s, x(s), u(s)) d s=\lim _{t \rightarrow \infty} \int_{t_{0}}^{t} L(s, x(s), u(s)) d s .
$$

We claim that (H1) iv), v) imply that the above limit does exist and belongs to $[-\infty, \infty)$. Indeed, since $L(s, x, u) \leq \alpha(s)$ for a.e. $s \geq S$ and all $x \in K$, $u \in U(s)$, the mapping $t \mapsto \int_{S}^{t}(L(s, x(s), u(s))-\alpha(s)) d s$ is nonincreasing on $[S, \infty)$ (with respect to $t$ ) and so it has a limit when $t \rightarrow \infty$ (possibly equal to $-\infty)$. Since

$$
\int_{S}^{t}(L(s, x(s), u(s))-\alpha(s)) d s=\int_{S}^{t} L(s, x(s), u(s)) d s-\int_{S}^{t} \alpha(s) d s \leq 0
$$

and $\lim _{t \rightarrow \infty} \int_{S}^{t} \alpha(s) d s$ does exist and is finite, it follows that also the limit $\lim _{t \rightarrow \infty} \int_{S}^{t} L(s, x(s), u(s)) d s$ does exist and is different from $+\infty$. On the other hand, by (H1) iv) the integral $\int_{t_{0}}^{S} L(s, x(s), u(s)) d s$ is finite, proving our claim.

For any $\left(t_{0}, x_{0}\right) \in \mathbb{R}_{+} \times K$, a feasible trajectory-control pair $(\bar{x}, \bar{u})$ on $\left[t_{0}, \infty\right)$ is called optimal for the infinite horizon problem at $\left(t_{0}, x_{0}\right)$ if $\bar{x}\left(t_{0}\right)=$ $x_{0}$ and for every feasible trajectory-control pair $(x, u)$ on $\left[t_{0}, \infty\right)$ satisfying $x\left(t_{0}\right)=x_{0}$ we have

$$
\int_{t_{0}}^{\infty} L(t, \bar{x}(t), \bar{u}(t)) d t \geq \int_{t_{0}}^{\infty} L(t, x(t), u(t)) d t .
$$

It is not difficult to realize that if (H1) i), iv), v) are satisfied, then $V$ is locally bounded from the above on $\operatorname{dom}(V)$ and that $V$ takes values in $[-\infty, \infty)$.

\section{UPPER SEMICONTINUITY OF THE VALUE FUNCTION}

The question of existence of optimal controls is pretty well understood. The standard proofs rely on taking limits of maximizing subsequences of trajectories and weak limits of their derivatives. Cesari-Olech type convexity and upper semicontinuity assumptions are needed to justify that the limiting trajectory is optimal. The very same arguments can be applied as well to study the upper semicontinuity of the value function. 
Theorem 4.1. Assume (H1). Then $V$ is upper semicontinuous, takes values in $[-\infty, \infty)$ and for every $\left(t_{0}, x_{0}\right) \in \operatorname{dom}(V)$, there exists a feasible trajectorycontrol pair $(\bar{x}, \bar{u})$ satisfying $V\left(t_{0}, x_{0}\right)=\int_{t_{0}}^{\infty} L(t, \bar{x}(t), \bar{u}(t)) d t$.

Moreover, if

$$
\left\{\begin{array}{l}
\exists \bar{S}>0 \text { and an integrable } \delta:[\bar{S}, \infty) \rightarrow \mathbb{R}_{+} \text {such that } \\
L(t, x, u) \geq-\delta(t) \text { for a.e. } t>\bar{S}, \forall x \in K, u \in U(t),
\end{array}\right.
$$

then

$$
\lim _{t \rightarrow \infty} \sup _{x \in \operatorname{dom}(V(t, \cdot))}|V(t, x)|=0 .
$$

Proof. Assumptions (H1) iv), v) and (7) yield that $V$ never takes value $+\infty$. The arguments for proving the existence of optimal solutions are well known. We recall them because similar ones will be also exploited in the other results of this paper.

Let $\left(t_{0}, x_{0}\right) \in \operatorname{dom}(V)$. Consider a maximizing sequence of feasible trajectorycontrol pairs $\left(x_{i}, u_{i}\right)$ satisfying $x_{i}\left(t_{0}\right)=x_{0}$. That is

$$
\lim _{i \rightarrow \infty} \int_{t_{0}}^{\infty} L\left(t, x_{i}(t), u_{i}(t)\right) d t=V\left(t_{0}, x_{0}\right)
$$

In particular, $x_{i}(t) \in K$ for all $t \geq t_{0}$ and $i \geq 1$. By (7), for every $T>t_{0}$, the restrictions of $x_{i}$ to $\left[t_{0}, T\right]$ are equibounded.

We construct the optimal trajectory control pair $(\bar{x}, \bar{u})$ of the infinite horizon problem using the induction argument. Let $R>0$ be such that for every $i$,

Then

$$
\sup _{t \in\left[t_{0}, t_{0}+1\right]}\left|x_{i}(t)\right| \leq R
$$

$$
\begin{aligned}
\left|f\left(t, x_{i}(t), u_{i}(t)\right)\right| & \leq\left|f\left(t, 0, u_{i}(t)\right)\right|+\left|f\left(t, x_{i}(t), u_{i}(t)\right)-f\left(t, 0, u_{i}(t)\right)\right| \\
& \leq c(t)+c_{R}(t) R
\end{aligned}
$$

Thus, (H1) iv) and the Dunford-Pettis theorem, imply that, taking a subsequence and keeping the same notation, we may assume that for some integrable functions $y:\left[t_{0}, t_{0}+1\right] \rightarrow \mathbb{R}^{n}, \gamma:\left[t_{0}, t_{0}+1\right] \rightarrow \mathbb{R}_{+}$, the restrictions of $\left(x_{i}^{\prime}(\cdot), L\left(\cdot, x_{i}(\cdot), u_{i}(\cdot)\right)\right)$ to $\left[t_{0}, t_{0}+1\right]$ converge weakly in $L^{1}\left(\left[t_{0}, t_{0}+1\right] ; \mathbb{R}^{n} \times \mathbb{R}\right)$ to $(y, \gamma)$. Define

$$
z_{i}(t)=\int_{t_{0}}^{t} L\left(t, x_{i}(t), u_{i}(t)\right) d t, \quad \forall t \in\left[t_{0}, t_{0}+1\right] .
$$

Since

$$
x_{i}(t)=x_{0}+\int_{t_{0}}^{t} f\left(t, x_{i}(t), u_{i}(t)\right) d t, \quad \forall t \in\left[t_{0}, t_{0}+1\right],
$$

we deduce that functions $\left(x_{i}, z_{i}\right)$ converge pointwise on $\left[t_{0}, t_{0}+1\right]$ when $i \rightarrow \infty$ to the function $(\bar{x}, z):\left[t_{0}, t_{0}+1\right] \rightarrow \mathbb{R}^{n} \times \mathbb{R}$ defined by

$$
\bar{x}(t)=x_{0}+\int_{t_{0}}^{t} y(t) d t, z(t)=\int_{t_{0}}^{t} \gamma(t) d t, \quad \forall t \in\left[t_{0}, t_{0}+1\right] .
$$


Hence $(\bar{x}, z)$ is absolutely continuous on $\left[t_{0}, t_{0}+1\right]$. Furthermore, $(7),(10)$, (H1) iv) and the Ascoli-Arzelà theorem imply that $\left(x_{i}, z_{i}\right)$ converge uniformly to $(\bar{x}, z)$. Moreover, at every Lebesgue point $t$ of $y(\cdot)$ we have $\bar{x}^{\prime}(t)=y(t)$.

Observe next that, by (H1) ii), for a.e. $t \in\left[t_{0}, t_{0}+1\right]$,

$$
\begin{aligned}
\left(x_{i}^{\prime}(t), L\left(t, x_{i}(t), u_{i}(t)\right)\right) \in & F\left(t, x_{i}(t)\right) \subset F(t, \bar{x}(t))+ \\
& \left(c_{R}(t)\left|x_{i}(t)-\bar{x}(t)\right|+\omega_{R}\left(t,\left|x_{i}(t)-\bar{x}(t)\right|\right)\right) B .
\end{aligned}
$$

Let $\varepsilon>0$ and $i_{0} \geq 1$ be such that $\sup _{t \in\left[t_{0}, t_{0}+1\right]}\left|x_{i}(t)-\bar{x}(t)\right| \leq \varepsilon$ for all $i \geq i_{0}$. The set

$$
F_{\varepsilon}(t, \bar{x}(t)):=F(t, \bar{x}(t))+\left(c_{R}(t) \varepsilon+\omega_{R}(t, \varepsilon)\right) B
$$

being convex and closed, also the set

$$
\mathcal{F}_{\varepsilon}:=\left\{(v, w) \in L^{1}\left(\left[t_{0}, t_{0}+1\right] ; \mathbb{R}^{n} \times \mathbb{R}\right) \mid(v(t), w(t)) \in F_{\varepsilon}(t, \bar{x}(t)) \text { for a.e. } t\right\}
$$

is convex and closed. Thus, by the Mazur theorem, it is weakly closed and therefore $\left(\bar{x}^{\prime}, \gamma\right) \in \mathcal{F}_{\varepsilon}$. So

$$
\left(\bar{x}^{\prime}(t), \gamma(t)\right) \in F(t, \bar{x}(t))+\left(c_{R}(t) \varepsilon+\omega_{R}(t, \varepsilon)\right) B \quad \text { for a.e. } t \in\left[t_{0}, t_{0}+1\right] .
$$

By the arbitrariness of $\varepsilon>0,\left(\bar{x}^{\prime}(t), \gamma(t)\right) \in F(t, \bar{x}(t))$ for a.e. $t \in\left[t_{0}, t_{0}+1\right]$.

From the measurable selection theorem [8, Theorem 8.2.10] we deduce that there exist a control $\bar{u}(\cdot)$ and a measurable function $r:\left[t_{0}, t_{0}+1\right] \rightarrow \mathbb{R}_{+}$ such that for a.e. $t \in\left[t_{0}, t_{0}+1\right]$,

$$
\bar{x}^{\prime}(t)=f(t, \bar{x}(t), \bar{u}(t)), \quad \gamma(t)=L(t, \bar{x}(t), \bar{u}(t))-r(t) .
$$

Since $x_{i}(t) \in K$ for all $t \geq t_{0}$ and $K$ is closed we know that $\bar{x}\left(\left[t_{0}, t_{0}+1\right]\right) \subset K$. Furthermore,

$$
\begin{aligned}
\lim _{i \rightarrow \infty} \int_{t_{0}}^{t_{0}+1} L\left(t, x_{i}(t), u_{i}(t)\right) d t & =\int_{t_{0}}^{t_{0}+1}(L(t, \bar{x}(t), \bar{u}(t))-r(t)) d t \\
& \left.\leq \int_{t_{0}}^{t_{0}+1} L(t, \bar{x}(t), \bar{u}(t))\right) d t .
\end{aligned}
$$

We extend next $(\bar{x}, \bar{u})$ on $\left[t_{0}, \infty\right)$. Set $\left(\bar{x}^{1}(t), \bar{u}^{1}(t)\right):=(\bar{x}(t), \bar{u}(t))$ for $t \in$ $\left[t_{0}, t_{0}+1\right]$. Let us assume that for some $k \geq 1$ we have constructed a subsequence $\left\{\left(x_{i_{j}}^{k}, u_{i_{j}}^{k}\right)\right\}_{j}$ of $\left\{\left(x_{i}, u_{i}\right)\right\}_{i}$, a trajectory control pair $\left(\bar{x}^{k}, \bar{u}^{k}\right)$ on $\left[t_{0}, t_{0}+k\right]$ and an absolutely continuous function $z^{k} \in W^{1,1}\left(\left[t_{0}, t_{0}+k\right] ; \mathbb{R}\right)$ such that $\bar{x}^{k}\left(\left[t_{0}, t_{0}+k\right]\right) \subset K$ and for

$$
z_{i_{j}}^{k}(t):=\int_{t_{0}}^{t} L\left(s, x_{i_{j}}^{k}(s), u_{i_{j}}^{k}(s)\right) d s, \quad \forall t \in\left[t_{0}, t_{0}+k\right]
$$

the following holds true :

$\left(x_{i_{j}}^{k}, z_{i_{j}}^{k}\right)$ converge uniformly on $\left[t_{0}, t_{0}+k\right]$ to $\left(\bar{x}^{k}, z^{k}\right),\left(\left(x_{i_{j}}^{k}\right)^{\prime},\left(z_{i_{j}}^{k}\right)^{\prime}\right)$ converge weakly in $L^{1}\left(\left[t_{0}, t_{0}+k\right] ; \mathbb{R}^{n} \times \mathbb{R}\right)$ to $\left(\left(\bar{x}^{k}\right)^{\prime},\left(z^{k}\right)^{\prime}\right)$ and if $k \geq 2$

$$
\left(\bar{x}^{k}(t), \bar{u}^{k}(t), z^{k}(t)\right)=\left(\bar{x}^{k-1}(t), \bar{u}^{k-1}(t), z^{k-1}(t)\right) \forall t \in\left[t_{0}, t_{0}+k-1\right] .
$$

Consider the interval $\left[t_{0}, t_{0}+k+1\right]$. By the same arguments, we find a subsequence $\left\{\left(x_{i_{j_{\ell}}}^{k}, u_{i_{j_{\ell}}}^{k}, z_{i_{j_{\ell}}}^{k}\right)\right\}_{\ell}$ of $\left\{\left(x_{i_{j}}^{k}, u_{i_{j}}^{k}, z_{i_{j}}^{k}\right)\right\}_{j}$, a trajectory-control pair $\left(\bar{x}^{k+1}, \bar{u}^{k+1}\right)$ on $\left[t_{0}, t_{0}+k+1\right]$ and an absolutely continuous $z^{k+1}:\left[t_{0}, t_{0}+\right.$ 
$k+1] \rightarrow \mathbb{R}$, such that $\left(x_{i_{j_{\ell}}}^{k}, z_{i_{j_{\ell}}}^{k}\right)$ converge uniformly on $\left[t_{0}, t_{0}+k+1\right]$ to $\left(\bar{x}^{k+1}, z^{k+1}\right),\left(\left(x_{i_{j_{\ell}}}^{k}\right)^{\prime},\left(z_{i_{j_{\ell}}}^{k}\right)^{\prime}\right)$ converge weakly in $L^{1}\left(\left[t_{0}, t_{0}+k+1\right] ; \mathbb{R}^{n} \times \mathbb{R}\right)$ to $\left(\left(\bar{x}^{k+1}\right)^{\prime},\left(z^{k+1}\right)^{\prime}\right)$,

$$
\lim _{\ell \rightarrow \infty} \int_{t_{0}}^{t_{0}+k+1} L\left(t, x_{i_{j_{\ell}}}^{k}(t), u_{i_{j_{\ell}}}^{k}(t)\right) d t \leq \int_{t_{0}}^{t_{0}+k+1} L\left(t, \bar{x}^{k+1}(t), \bar{u}^{k+1}(t)\right) d t
$$

and $\bar{x}^{k+1}\left(\left[t_{0}, t_{0}+k+1\right]\right) \subset K$,

$$
\left(\bar{x}^{k+1}(t), \bar{u}^{k+1}(t), z^{k+1}(t)\right)=\left(\bar{x}^{k}(t), \bar{u}^{k}(t), z^{k}(t)\right) \forall t \in\left[t_{0}, t_{0}+k\right] .
$$

Rename $\left(x_{i_{j_{\ell}}}^{k}, u_{i_{j_{\ell}}}^{k}\right)$ by $\left(x_{i_{j}}^{k+1}, u_{i_{j}}^{k+1}\right)$ and set $(\bar{x}(t), \bar{u}(t))=\left(\bar{x}^{k+1}(t), \bar{u}^{k+1}(t)\right)$ for $t \in\left[t_{0}, t_{0}+k+1\right]$. Applying the induction argument with respect to $k$ we obtain a trajectory-control pair $(\bar{x}, \bar{u})$ defined on $\left[t_{0}, \infty\right)$.

To show that it is optimal, fix $\varepsilon>0$. By H1) v) for all large $T$ and for every feasible trajectory-control pair $(x, u)$ on $\left[t_{0}, \infty\right)$,

$$
\int_{T}^{\infty} L(s, x(s), u(s)) d s \leq \varepsilon .
$$

Consequently for any fixed sufficiently large $k$, using the same notation as before, we get

$$
\begin{gathered}
\lim _{j \rightarrow \infty} \int_{t_{0}}^{\infty} L\left(t, x_{i_{j}}^{k}(t), u_{i_{j}}^{k}(t)\right) d t \leq \lim _{j \rightarrow \infty} \int_{t_{0}}^{t_{0}+k} L\left(t, x_{i_{j}}^{k}(t), u_{i_{j}}^{k}(t)\right) d t+\varepsilon \\
\leq \int_{t_{0}}^{t_{0}+k} L(t, \bar{x}(t), \bar{u}(t)) d t+\varepsilon .
\end{gathered}
$$

We proved that for every $\varepsilon>0$ and all large $k$,

$$
V\left(t_{0}, x_{0}\right) \leq \int_{t_{0}}^{t_{0}+k} L(t, \bar{x}(t), \bar{u}(t)) d t+\varepsilon
$$

Taking the limit when $k \rightarrow \infty$ we obtain

$$
V\left(t_{0}, x_{0}\right) \leq \int_{t_{0}}^{\infty} L(t, \bar{x}(t), \bar{u}(t)) d t+\varepsilon .
$$

Since $\varepsilon>0$ is arbitrary, this inequality implies that $(\bar{x}, \bar{u})$ is optimal.

To prove the upper semicontinuity of $V$, consider a sequence $\left(t_{0}^{i}, x_{0}^{i}\right) \in$ $\mathbb{R}_{+} \times K$ converging to some $\left(t_{0}, x_{0}\right)$ when $i \rightarrow \infty$. We have to show that $\limsup _{i \rightarrow \infty} V\left(t_{0}^{i}, x_{0}^{i}\right) \leq V\left(t_{0}, x_{0}\right)$. If for all large $i, V\left(t_{0}^{i}, x_{0}^{i}\right)=-\infty$, then we are done. So it is enough to consider the case when $\left(t_{0}^{i}, x_{0}^{i}\right) \in \operatorname{dom}(V)$ for all $i$.

Let $\left(x_{i}, u_{i}\right)$ be an optimal trajectory-control pair corresponding to the initial condition $\left(t_{0}^{i}, x_{0}^{i}\right)$. If $t_{0}^{i}>t_{0}$, then we extend $x_{i}$ on $\left[t_{0}, t_{0}^{i}\right]$ by setting $x_{i}(s)=x_{0}^{i}$ for $s \in\left[t_{0}, t_{0}^{i}\right]$. Using exactly the same arguments as before we construct a trajectory-control pair $(\bar{x}, \bar{u})$ such that $\bar{x}\left(t_{0}\right)=x_{0}$ and for every $\varepsilon>0$ and all large $k$,

$$
\limsup _{i \rightarrow \infty} \int_{t_{i}}^{\infty} L\left(t, x_{i}(t), u_{i}(t)\right) d t \leq \int_{t_{0}}^{t_{0}+k} L(t, \bar{x}(t), \bar{u}(t)) d t+\varepsilon .
$$


Taking the limit when $k \rightarrow \infty$ and using that $\varepsilon$ is arbitrary, the upper semicontinuity of $V$ at $\left(t_{0}, x_{0}\right)$ follows.

Suppose next that (8) is satisfied. Thus, if $t$ is sufficiently large and $x_{t} \in \operatorname{dom}(V(t, \cdot))$, then $\left.\mid V\left(t, x_{t}\right)\right) \mid \leq \int_{t}^{\infty}(\alpha(s)+\delta(s)) d s$. Then (9) follows from the equality $\lim _{t \rightarrow \infty} \int_{t}^{\infty}(\alpha(s)+\delta(s)) d s=0$.

In the above proof we needed assumption (H1) i) to deduce (7). A different assumption involving bounds on the growth of $|f|$ with respect to $L$ is convenient as well. Its advantage lies in the fact that we do not request anymore the sets $f(t, x, U(t))$ to be bounded.

Theorem 4.2. Assume (H1) ii)-vi) and (8). If there exist $c>0, r>0$ and $\theta \in L_{\mathrm{loc}}^{1}\left(\mathbb{R}_{+} ; \mathbb{R}_{+}\right)$such that for a.e. $t \geq 0$,

$$
|f(t, x, u)|^{1+r} \leq \theta(t)+c L(t, x, u) \forall x \in \mathbb{R}^{n}, u \in U(t),
$$

then (9) holds true and for every $\left(t_{0}, x_{0}\right) \in \operatorname{dom}(V)$, there exists a feasible trajectory-control pair $(\bar{x}, \bar{u})$ satisfying $V\left(t_{0}, x_{0}\right)=\int_{t_{0}}^{\infty} L(t, \bar{x}(t), \bar{u}(t)) d t$.

Furthermore, if $V$ is locally bounded from the above, then it is upper semicontinuous.

Proof. Conclusion (9) follows as in the proof of Theorem 4.1. Let $\left(t_{0}, x_{0}\right) \in$ $\operatorname{dom}(V)$. Consider a maximizing sequence of feasible trajectory-control pairs $\left(x_{i}, u_{i}\right)$ satisfying $x_{i}\left(t_{0}\right)=x_{0}$. By our assumptions, for a.e. $t \geq t_{0}$ and all $i \geq 1$,

$$
\left|f\left(t, x_{i}(t), u_{i}(t)\right)\right|^{1+r} \leq \theta(t)+c L\left(t, x_{i}(t), u_{i}(t)\right) .
$$

Hence for every $t \geq t_{0}$,

$$
\sup _{i \geq 1} \int_{t_{0}}^{t}\left|f\left(s, x_{i}(s), u_{i}(s)\right)\right|^{1+r} d s \leq \sup _{i \geq 1} \int_{t_{0}}^{t}\left(\theta(s)+c L\left(s, x_{i}(s), u_{i}(s)\right)\right) d s .
$$

Observe next that there exists $M>0$ such that for all large $t>t_{0}$ and every $i$,

$$
\int_{t_{0}}^{t} L\left(s, x_{i}(s), u_{i}(s)\right) d s \leq \int_{t_{0}}^{\infty} L\left(s, x_{i}(s), u_{i}(s)\right) d s+\int_{t}^{\infty} \delta(s) d s<M .
$$

Consequently $\left\{f\left(\cdot, x_{i}(\cdot), u_{i}(\cdot)\right)\right\}_{i}$ is bounded in $L^{1+r}\left(\left[t_{0}, t\right] ; \mathbb{R}^{n}\right)$ and therefore it is also bounded in $L^{1+r}\left(\left[t_{0}, t_{0}+1\right] ; \mathbb{R}^{n}\right)$. Since $L^{1+r}\left(\left[t_{0}, t_{0}+1\right] ; \mathbb{R}^{n}\right)$ is reflexive, taking a subsequence and keeping the same notation, we may assume that for some integrable function $y:\left[t_{0}, t_{0}+1\right] \rightarrow \mathbb{R}^{n}$, the restrictions of $x_{i}^{\prime}(\cdot)$ to $\left[t_{0}, t_{0}+1\right]$ converge weakly in $L^{1+r}\left(\left[t_{0}, t_{0}+1\right] ; \mathbb{R}^{n}\right)$ to $y$.

Set $p=(1+r) / r$. By the Hölder inequality, for any $i$ and for all $t_{0} \leq a<$ $b \leq t_{0}+1$,

$$
\left|x_{i}(b)-x_{i}(a)\right| \leq(b-a)^{\frac{1}{p}}\left(\int_{a}^{b}\left|f\left(s, x_{i}(s), u_{i}(s)\right)\right|^{1+r} d s\right)^{\frac{1}{1+r}} .
$$

Therefore $\left\{x_{i}\right\}_{i}$ are equicontinuous on $\left[t_{0}, t_{0}+1\right]$. Starting at this point the same arguments as those in the proof of Theorem 4.1 can be applied to 
get the existence of an optimal solution at the initial condition $\left(t_{0}, x_{0}\right)$. The upper semicontinuity of $V$ can be proved in a similar way using that $V$ is locally bounded from the above.

\section{Relaxation in the absence of State COnstraints}

In the previous section we have shown that the value function is upper semicontinuous and that the optimal trajectories do exist assuming that the sets $F(t, x)$ are closed and convex. If the convexity assumption (H1) vi) is not imposed, then, in the literature, one usually considers the so-called relaxed problems.

In this section we restrict our attention to the case when $K=\mathbb{R}^{n}$, that is without state constraints. Consider the relaxed infinite horizon problem

$$
V^{r e l}\left(t_{0}, x_{0}\right)=\sup \int_{t_{0}}^{\infty}\left(\sum_{i=0}^{n} \lambda_{i}(t) L\left(t, x(t), u_{i}(t)\right)\right) d t
$$

over all trajectory-control pairs of

$$
\left\{\begin{array}{l}
x^{\prime}(t)=\sum_{i=0}^{n} \lambda_{i}(t) f\left(t, x(t), u_{i}(t)\right), u_{i}(t) \in U(t), \lambda_{i}(t) \geq 0, \sum_{i=0}^{n} \lambda_{i}(t)=1 \\
x\left(t_{0}\right)=x_{0},
\end{array}\right.
$$

where $u_{i}(\cdot), \lambda_{i}(\cdot)$ are Lebesgue measurable on $\mathbb{R}_{+}$for $i=0, \ldots, n$. Then $V^{r e l} \geq V$. For $v=\left(u_{0}, \ldots, u_{n}\right), \Lambda=\left(\lambda_{0}, \ldots, \lambda_{n}\right)$ define

$$
\hat{f}(t, x, v, \Lambda)=\sum_{i=0}^{n} \lambda_{i} f\left(t, x, u_{i}\right), \quad \hat{L}(t, x, v, \Lambda)=\sum_{i=0}^{n} \lambda_{i} L\left(t, x, u_{i}\right)
$$

and

$$
\widehat{U}(t):=\underbrace{U(t) \times \ldots \times U(t)}_{n+1} \times\left\{\left(\lambda_{0}, \ldots, \lambda_{n}\right) \mid \lambda_{i} \geq 0 \forall i, \Sigma_{i=0}^{n} \lambda_{i}=1\right\} .
$$

Thus the relaxed problem is of type (1) - (2) with $f, L$ replaced by $\hat{f}, \hat{L}$ and $U(t)$ replaced by $\widehat{U}(t)$.

Our first relaxation result addresses a case where the celebrated FilippovWażewski theorem can be applied.

Theorem 5.1. Assume (H1) $i)-v$ ) with $\omega_{R}(t, r)=\bar{c}_{R}(t) r$, where for all $R>0, \bar{c}_{R}: \mathbb{R}_{+} \rightarrow \mathbb{R}_{+}$is locally integrable, and that for a.e. $t \in \mathbb{R}_{+}$and all $x \in \mathbb{R}^{n}$, the set

$$
G(t, x):=\{(f(t, x, u), L(t, x, u)): u \in U(t)\}
$$

is compact. Further assume that (8) is satisfied.

Then $V^{r e l}=V$ on $\mathbb{R}_{+} \times \mathbb{R}^{n}$ and for every $\left(t_{0}, x_{0}\right) \in \mathbb{R}_{+} \times \mathbb{R}^{n}$, there exists $\left(\bar{x}(\cdot), \bar{v}(\cdot)=\left(\bar{u}_{0}(\cdot), \ldots, \bar{u}_{n}(\cdot)\right), \bar{\Lambda}(\cdot)=\left(\bar{\lambda}_{0}(\cdot), \ldots, \bar{\lambda}_{n}(\cdot)\right)\right)$ satisfying (13) such that

$$
V^{r e l}\left(t_{0}, x_{0}\right)=\int_{t_{0}}^{\infty} \hat{L}(t, \bar{x}(t), \bar{v}(t), \bar{\Lambda}(t)) d t .
$$


Furthermore, (9) holds true.

In particular, if a trajectory-control pair $(\bar{x}, \bar{u})$ is optimal for (1), (2), then it is also optimal for the relaxed problem (12), (13).

\section{Remark 5.2.}

a) If the set $U(t)$ is compact, then so is $G(t, x)$.

b) In [14] a similar relaxation result was proved under slightly different assumptions. It can be shown that (8) and (H1) $i)-v)$ imply that $V^{r e l}(t, \cdot)$ is continuous and so the proof could be done using the same scheme as in [14]. However, as we show below, under our assumptions it can be simplified avoiding the use of $V^{r e l}$.

Proof. It is not difficult to realize that $\hat{f}, \hat{L}, \widehat{U}$ satisfy $(\mathrm{H} 1)$. Let $\left(t_{0}, x_{0}\right) \in$ $\mathbb{R}_{+} \times \mathbb{R}^{n}$. Since $K=\mathbb{R}^{n}$, Theorem 4.1 and (8) imply that $V^{r e l} \neq \pm \infty$. By Theorem 4.1 applied to $\hat{f}, \hat{L}, \widehat{U}$, there exists $(\bar{x}(\cdot), \bar{v}(\cdot), \bar{\Lambda}(\cdot))$ satisfying (13) such that

$$
V^{r e l}\left(t_{0}, x_{0}\right)=\int_{t_{0}}^{\infty} \hat{L}(t, \bar{x}(t), \bar{v}(t), \bar{\Lambda}(t)) d t .
$$

Fix $\varepsilon>0$ and let $k>\max \left\{t_{0}, S, \bar{S}\right\}$ be so that $\int_{k}^{\infty}(\alpha(t)+\delta(t)) d t \leq \varepsilon / 3$.

Then

$$
\begin{aligned}
V^{r e l}\left(t_{0}, x_{0}\right) & =\int_{k}^{\infty} \hat{L}(t, \bar{x}(t), \bar{v}(t), \bar{\Lambda}(t)) d t+\int_{t_{0}}^{k} \hat{L}(t, \bar{x}(t), \bar{v}(t), \bar{\Lambda}(t)) d t \\
& \leq \frac{\varepsilon}{3}+\int_{t_{0}}^{k} \hat{L}(t, \bar{x}(t), \bar{v}(t), \bar{\Lambda}(t)) d t .
\end{aligned}
$$

By the Filippov-Ważewski relaxation theorem and the measurable selection theorem, see for instance [24], there exists $u_{\varepsilon} \in \mathcal{U}$ such that the solution $x_{\varepsilon}$ of the system

$$
x^{\prime}=f\left(t, x, u_{\varepsilon}(t)\right), x\left(t_{0}\right)=x_{0}
$$

satisfies

$$
\left|\int_{t_{0}}^{k} \hat{L}(t, \bar{x}(t), \bar{v}(t), \bar{\Lambda}(t)) d t-\int_{t_{0}}^{k} L\left(t, x_{\varepsilon}(t), u_{\varepsilon}(t)\right) d t\right|<\frac{\varepsilon}{3} .
$$

Consider any trajectory-control pair $(x, u)$ of $(5)$ on $[k, \infty)$ with $x(k)=x_{\varepsilon}(k)$. Thus

$$
\int_{k}^{\infty} L(t, x(t), u(t)) d t \geq \int_{k}^{\infty}(-\delta(t)) d t
$$

We extend the trajectory-control pair $\left(x_{\varepsilon}, u_{\varepsilon}\right)$ on the time interval $[k, \infty)$ by setting $\left(x_{\varepsilon}(s), u_{\varepsilon}(s)\right)=(x(s), u(s))$ for $s>k$. Hence

$$
\begin{aligned}
V^{r e l}\left(t_{0}, x_{0}\right) & \leq \frac{2 \varepsilon}{3}+\int_{t_{0}}^{k} L\left(t, x_{\varepsilon}(t), u_{\varepsilon}(t)\right) d t \\
& \leq \frac{2 \varepsilon}{3}+\int_{t_{0}}^{\infty} L\left(t, x_{\varepsilon}(t), u_{\varepsilon}(t)\right) d t+\int_{k}^{\infty} \delta(t) d t \leq \varepsilon+V\left(t_{0}, x_{0}\right) .
\end{aligned}
$$

This yields $V^{r e l}\left(t_{0}, x_{0}\right) \leq V\left(t_{0}, x_{0}\right)+\varepsilon$. Since $\varepsilon>0$ and $\left(t_{0}, x_{0}\right)$ are arbitrary, we get $V^{r e l}=V$. 
The above result has a restrictive assumption of compactness of sets $G(t, x)$ because in the proof we used the Filippov-Ważewski relaxation theorem dealing with compact valued maps. In the case of control systems this theorem can be stated without such compactness assumption.

Theorem 5.3 (Finite horizon relaxation theorem). Let $T>0, U:[0, T] \leadsto$ $\mathbb{R}^{m}$ be measurable, with closed nonempty images, $g:[0, T] \times \mathbb{R}^{n} \times \mathbb{R}^{m} \rightarrow \mathbb{R}^{n}$ be a Carathéodory function such that for every $R>0$, there exists $c_{R} \in$ $L^{1}\left([0, T] ; \mathbb{R}_{+}\right)$satisfying for a.e. $t \in[0, T]$,

$$
|g(t, x, u)-g(t, y, u)| \leq c_{R}(t)|x-y| \forall x, y \in B(0, R), u \in U(t) .
$$

Further assume that $\psi(t):=\inf _{u \in U(t)}|g(t, 0, u)|$ is integrable on $[0, T]$.

Then for any $\varepsilon>0$ and any absolutely continuous $x:[0, T] \rightarrow \mathbb{R}^{n}$ satisfying

$$
x^{\prime}(t) \in \overline{c o} g(t, x(t), U(t)) \quad \text { a.e. in }[0, T],
$$

there exists a measurable selection $u(t) \in U(t)$ for $t \in[0, T]$ and an absolutely continuous function $x_{\varepsilon}:[0, T] \rightarrow \mathbb{R}^{n}$ such that

$$
x_{\varepsilon}^{\prime}(t)=g\left(t, x_{\varepsilon}(t), u(t)\right) \text { a.e. in }[0, T], \quad x_{\varepsilon}(0)=x(0)
$$

and $\max _{t \in[0, T]}\left|x_{\varepsilon}(t)-x(t)\right|<\varepsilon$.

Remark 5.4. We would like to underline that in the above theorem the sets $g(t, x, U(t))$ are neither closed nor bounded.

Proof. We first observe that $g(t, 0, U(t)) \cap B(0, \psi(t)+\varepsilon) \neq \emptyset$ for every $\varepsilon>0$ and every $t \in[0, T]$. By the inverse image theorem [8, Theorem 8.2.9], there exists a measurable selection $u_{0}(t) \in U(t)$ for $t \in[0, T]$ such that $\left|g\left(t, 0, u_{0}(t)\right)\right| \leq \psi(t)+\varepsilon$ a.e. Thus the function $k:[0, T] \rightarrow \mathbb{R}_{+}$defined by $k(t):=\left|g\left(t, 0, u_{0}(t)\right)\right|$ is integrable. By the Castaing representation theorem, see for instance [8], there exist measurable selections $u_{i}(t) \in U(t), i \geq 1$ for $t \in[0, T]$ such that $U(t)=\overline{\bigcup_{i \geq 1}\left\{u_{i}(t)\right\}}$ for every $t \in[0, T]$. Fix an integer $j \geq 1$ and define

$$
\begin{gathered}
u_{i j}(t)=\left\{\begin{array}{l}
u_{i}(t) \quad \text { if } \\
u_{0}(t) \quad \text { otherwise, }
\end{array}\right. \\
U_{j}(t)=\left\{u_{0}(t)\right\} \cup \bigcup_{1 \leq i \leq j}\left\{u_{i j}(t)\right\} .
\end{gathered}
$$

Observe that for every $t$, the family of finite sets $U_{j}(t)$ is increasing (with respect to $j$ ) and $U(t)=\overline{\bigcup_{j \geq 1} U_{j}(t)}$. By the continuity of $g(t, x, \cdot)$, for a.e. $t \in[0, T]$ and all $x \in \mathbb{R}^{n}$,

$$
\overline{\bigcup_{j \geq 1} \operatorname{cog}\left(t, x, U_{j}(t)\right)}=\overline{c o} g(t, x, U(t)) .
$$

Notice that for every $t$, the set $g\left(t, x, U_{j}(t)\right)$ is compact. Let $x(\cdot) \in$ $W^{1,1}\left([0, T] ; \mathbb{R}^{n}\right)$ satisfy $(14)$. Then

$$
\begin{aligned}
\gamma_{j}(t) & :=d_{\operatorname{cog}\left(t, x(t), U_{j}(t)\right)}\left(x^{\prime}(t)\right) \leq\left|x^{\prime}(t)-g\left(t, 0, u_{0}(t)\right)\right|+c_{r}(t)|x(t)| \\
& \leq\left|x^{\prime}(t)\right|+\left|g\left(t, 0, u_{0}(t)\right)\right|+c_{r}(t)|x(t)|,
\end{aligned}
$$


where $r>0$ is so that $\max _{t \in[0, T]}|x(t)|<r$. Hence $\left\{\gamma_{j}\right\}_{j \geq 1}$ are bounded by an integrable function. Moreover $\lim _{j \rightarrow \infty} \gamma_{j}(t)=0$ for a.e. $t \in[0, T]$. Let

$$
t_{j}=\max \left\{t \in[0, T]: e^{\int_{0}^{T} c_{2 r}(s) d s} \int_{0}^{t} \gamma_{j}(s) d s \leq r\right\} .
$$

Since $\int_{0}^{T} \gamma_{j}(s) d s$ converge to zero when $j \rightarrow \infty$, we deduce that $t_{j}=T$ for all $j$ larger than some $j_{0}$. By the Filippov theorem, see for instance [24, Theorem 1.2] and the remark following it, for every $j \geq j_{0}$, there exists an absolutely continuous function $x_{j}:[0, T] \rightarrow \mathbb{R}^{n}$ such that $x_{j}^{\prime}(t) \in \operatorname{cog}\left(t, x_{j}(t), U_{j}(t)\right)$ a.e. in $[0, T], x_{j}(0)=x(0)$ and

$$
\sup _{t \in[0, T]}\left|x_{j}(t)-x(t)\right| \leq e^{\int_{0}^{T} c_{2 r}(s) d s} \int_{0}^{T} \gamma_{j}(s) d s .
$$

Let $\varepsilon>0$ and consider $j \geq j_{0}$ such that $e^{\int_{0}^{T} c_{2 r}(s) d s} \int_{0}^{T} \gamma_{j}(s) d s<\frac{\varepsilon}{2}$. By the Filippov-Ważewski relaxation theorem there exists an absolutely continuous function $x_{\varepsilon}:[0, T] \rightarrow \mathbb{R}^{n}$ such that

$$
x_{\varepsilon}^{\prime}(t) \in g\left(t, x_{\varepsilon}(t), U_{j}(t)\right) \text { a.e. in }[0, T], x_{\varepsilon}(0)=x(0)
$$

and $\sup _{t \in[0, T]}\left|x_{\varepsilon}(t)-x_{j}(t)\right|<\varepsilon / 2$. By the measurable selection theorem we can find a measurable selection $u_{\varepsilon}(t) \in U_{j}(t)$ such that for a.e. $t \in[0, T]$ we have $x_{\varepsilon}^{\prime}(t)=g\left(t, x_{\varepsilon}(t), u_{\varepsilon}(t)\right)$. Since

$$
\sup _{t \in[0, T]}\left|x_{\varepsilon}(t)-x(t)\right| \leq \sup _{t \in[0, T]}\left(\left|x_{j}(t)-x(t)\right|+\left|x_{\varepsilon}(t)-x_{j}(t)\right|\right)<\varepsilon
$$

the proof is complete.

We next apply the above result to the infinite horizon relaxation problem with possibly unbounded and not necessarily closed sets $f(t, x, U(t))$. Theorems 5.5 and 5.7 below are new.

Theorem 5.5. Assume (H1) ii) $-v$ ) with $\omega_{R}(t, r)=\bar{c}_{R}(t) r$, where for all $R>0, \bar{c}_{R}: \mathbb{R}_{+} \rightarrow \mathbb{R}_{+}$is locally integrable, and that for some $T_{0}>0$ and all $t_{0} \geq T_{0}, x_{0} \in \mathbb{R}^{n}$ there exists a trajectory of (2) defined on $\left[t_{0}, \infty\right)$. Further assume that (8) holds true and that the function

$$
\psi(t):=\inf \{|f(t, 0, u)|+|L(t, 0, u)|: u \in U(t)\}
$$

is locally integrable on $\mathbb{R}_{+}$. Then (9) is satisfied and $V^{r e l}=V$.

Remark 5.6. The assumption that for some $T_{0}>0$ and all $t_{0} \geq T_{0}, x_{0} \in \mathbb{R}^{n}$ there exists a trajectory of (2) defined on $\left[t_{0}, \infty\right)$ holds true, for instance, whenever the function $\psi(\cdot)$ is locally integrable on $\mathbb{R}_{+}$and there exists $T_{0}>0$ such that $c_{R}(t)$ do not depend on $R$ for all $t \geq T_{0}$. Indeed, by the proof of Theorem 5.3, we know that there exists a measurable selection $u_{0}(t) \in U(t)$ such that $\left|f\left(t, 0, u_{0}(t)\right)\right| \leq \psi(t)+1$ for every $t \in \mathbb{R}_{+}$. Furthermore, setting 
$c(t)=c_{R}(t)$ for $t \geq T_{0}$ we obtain $\left|f\left(t, x, u_{0}(t)\right)\right| \leq \psi(t)+1+c(t)|x|$. Given $t_{0} \geq T_{0}, x_{0} \in \mathbb{R}^{n}$, it is enough to consider the solution of

$$
x^{\prime}=f\left(t, x, u_{0}(t)\right), x\left(t_{0}\right)=x_{0} .
$$

Proof. (of Theorem 5.5). Clearly $V \leq V^{r e l}$. Therefore, if $V^{r e l}\left(t_{0}, x_{0}\right)=-\infty$, then $V\left(t_{0}, x_{0}\right)=-\infty$. We first show that for every $\left(t_{0}, x_{0}\right) \in \operatorname{dom}\left(V^{r e l}\right)$ we have $V^{\text {rel }}\left(t_{0}, x_{0}\right) \leq V\left(t_{0}, x_{0}\right)$. Fix $\left(t_{0}, x_{0}\right) \in \operatorname{dom}\left(V^{\text {rel }}\right), \varepsilon>0$ and consider a trajectory-control pair $(x, v, \Lambda)$ of the relaxed system satisfying $V^{r e l}\left(t_{0}, x_{0}\right) \leq$ $\int_{t_{0}}^{\infty} \hat{L}(t, x(t), v(t), \Lambda(t)) d t+\frac{\varepsilon}{4}$.

By our assumptions, for every sufficiently large $k>0$ and any trajectorycontrol pair $(\tilde{x}, \tilde{u})$ defined on $[k, \infty)$, we have

$$
\int_{k}^{\infty}|L(t, \tilde{x}(t), \tilde{u}(t))| d t \leq \frac{\varepsilon}{4}, \int_{k}^{\infty}|\hat{L}(t, x(t), v(t), \Lambda(t))| d t \leq \frac{\varepsilon}{4} .
$$

By Theorem 5.3 applied to the function $g(t, x, u)=(f(t, x, u), L(t, x, u))$ and the time interval $\left[t_{0}, k\right]$ with $k>t_{0}$, there exists a trajectory-control pair $\left(x_{\varepsilon}, u_{\varepsilon}\right)$ of the control system (2) defined on $\left[t_{0}, k\right]$ such that

$$
\left|\int_{t_{0}}^{k} \hat{L}(t, x(t), v(t), \Lambda(t)) d t-\int_{t_{0}}^{k} L\left(t, x_{\varepsilon}(t), u_{\varepsilon}(t)\right) d t\right|<\frac{\varepsilon}{4} .
$$

Consider $k>0$ sufficiently large and a trajectory-control pair $(x, u)$ of $(5)$ defined on $[k, \infty)$ and satisfying $x(k)=x_{\varepsilon}(k)$. Set $\left(x_{\varepsilon}(t), u_{\varepsilon}(t)\right)=(x(t), u(t))$ for $t>k$. Then

$$
\begin{aligned}
& V^{r e l}\left(t_{0}, x_{0}\right) \leq \int_{t_{0}}^{\infty} \hat{L}(t, x(t), v(t), \Lambda(t)) d t+\frac{\varepsilon}{4} \leq \int_{t_{0}}^{k} \hat{L}(t, x(t), v(t), \Lambda(t)) d t+\frac{\varepsilon}{2} \\
& \leq \int_{t_{0}}^{k} L\left(t, x_{\varepsilon}(t), u_{\varepsilon}(t)\right) d t+\frac{3 \varepsilon}{4} \leq \int_{t_{0}}^{\infty} L\left(t, x_{\varepsilon}(t), u_{\varepsilon}(t)\right) d t+\varepsilon \leq V\left(t_{0}, x_{0}\right)+\varepsilon
\end{aligned}
$$

Hence $V^{r e l}\left(t_{0}, x_{0}\right) \leq V\left(t_{0}, x_{0}\right)$, by the arbitrariness of $\varepsilon>0$.

It remains to consider the case $V^{r e l}\left(t_{0}, x_{0}\right)=+\infty$. Then there exist trajectory-control pairs $\left(x^{i}, v^{i}, \Lambda^{i}\right)$ of the relaxed problem such that

$$
\lim _{i \rightarrow \infty} \int_{t_{0}}^{\infty} \hat{L}\left(t, x^{i}(t), v^{i}(t), \Lambda^{i}(t)\right) d t=+\infty .
$$

Applying the same arguments as before, for every $k>0$ and $\varepsilon>0$, we can find trajectory-control pairs $\left(x_{\varepsilon}^{i}, u_{\varepsilon}^{i}\right)$ of $(5)$ defined on $\left[t_{0}, \infty\right)$, satisfying $x_{\varepsilon}^{i}\left(t_{0}\right)=x_{0}$ and

$$
\left|\int_{t_{0}}^{k} \hat{L}\left(t, x^{i}(t), v^{i}(t), \Lambda^{i}(t)\right) d t-\int_{t_{0}}^{k} L\left(t, x_{\varepsilon}^{i}(t), u_{\varepsilon}^{i}(t)\right) d t\right|<\frac{\varepsilon}{3} .
$$

Moreover, for $k>0$ large enough and every $i$ we have

$$
\int_{k}^{\infty}\left|\hat{L}\left(t, x^{i}(t), v^{i}(t), \Lambda^{i}(t)\right)\right| d t<\frac{\varepsilon}{3}, \quad \int_{k}^{\infty}\left|L\left(t, x_{\varepsilon}^{i}(t), u_{\varepsilon}^{i}(t)\right)\right| d t<\frac{\varepsilon}{3} .
$$

Combining the above inequalities we get

$$
\int_{t_{0}}^{\infty} \hat{L}\left(t, x^{i}(t), v^{i}(t), \Lambda^{i}(t)\right) d t<\int_{t_{0}}^{\infty} L\left(t, x_{\epsilon}^{i}(t), u_{\varepsilon}^{i}(t)\right) d t+\varepsilon .
$$


Hence

$$
\lim _{i \rightarrow \infty} \int_{t_{0}}^{\infty} L\left(t, x_{\epsilon}^{i}(t), u_{\varepsilon}^{i}(t)\right) d t=+\infty
$$

and therefore $V\left(t_{0}, x_{0}\right)=+\infty$.

Finally we would like to observe that, in general, when the sets $G(t, x)$ are not compact, the sets $c o G(t, x)$ may be not closed. For this reason optimal solutions of the relaxed problem (12), (13) may not exist. To get the existence, without changing the value function, the correct relaxed problem in the case of unbounded, not necessarily closed sets $G(t, x)$, takes the following less familiar form. Define

$$
\bar{V}^{r e l}\left(t_{0}, x_{0}\right)=\sup \int_{t_{0}}^{\infty} \ell(t) d t
$$

over all $x \in W_{\text {loc }}^{1,1}\left(\left[t_{0}, \infty\right) ; \mathbb{R}^{n}\right)$ and $\ell \in L_{\text {loc }}^{1}\left(\left[t_{0}, \infty\right) ; \mathbb{R}\right)$ satisfying

$$
\left\{\begin{array}{l}
\left(x^{\prime}(t), \ell(t)\right) \in \overline{c o} G(t, x(t)) \text { a.e. in }\left[t_{0}, \infty\right) \\
x\left(t_{0}\right)=x_{0} .
\end{array}\right.
$$

Such a pair $(x, \ell)$ will be called below a solution of $(15)$. In the above we set $\bar{V}^{r e l}\left(t_{0}, x_{0}\right)=-\infty$ if $(15)$ does not have solutions defined on $\left[t_{0}, \infty\right)$.

Theorem 5.7. Under all the assumptions of Theorem 5.5 suppose that there exist $c>0, r>0$ and $\theta \in L_{\mathrm{loc}}^{1}\left([0, \infty) ; \mathbb{R}_{+}\right)$such that (11) holds true. Then for every $\left(t_{0}, x_{0}\right) \in \operatorname{dom}\left(\bar{V}^{\text {rel }}\right)$, there exists a solution $(\bar{x}(\cdot), \bar{\ell}(\cdot))$ of $(15)$ satisfying

$$
\bar{V}^{r e l}\left(t_{0}, x_{0}\right)=\int_{t_{0}}^{\infty} \bar{\ell}(t) d t .
$$

Furthermore, $\bar{V}^{\text {rel }}=V$ and (9) holds true. Moreover, if $V$ is locally bounded from the above, then it is upper semicontinuous.

Remark 5.8. Theorem 5.7 allows to avoid assumption (H1) vi) to claim that $V$ is upper semicontinuous and satisfies (9).

Proof. By our assumptions, $(\hat{f}, \hat{L}, \widehat{U})$ satisfy (11). Thus for every $(y, \ell) \in$ $\operatorname{co} G(t, x)$ we have $|y|^{1+r} \leq \theta(t)+c \ell$. Then the same inequality holds true also for any $(y, \ell) \in \overline{c o} G(t, x)$.

The same arguments as those in the proof of Theorem 4.2 imply (9) and that for every $\left(t_{0}, x_{0}\right) \in \operatorname{dom}\left(\bar{V}^{r e l}\right)$, there exists a solution $(\bar{x}(\cdot), \bar{\ell}(\cdot))$ of (15) satisfying (16). Also, as before, if $\bar{V}^{\text {rel }}$ is locally bounded from the above, then it is upper semicontinuous. It remains to show that $\bar{V}^{r e l}=V$. By Theorem 5.5 it is sufficient to verify that $\bar{V}^{\text {rel }}=V^{r e l}$. Fix $\left(t_{0}, x_{0}\right) \in$ $\mathbb{R}_{+} \times \mathbb{R}^{n}$. If $\bar{V}^{r e l}\left(t_{0}, x_{0}\right)=-\infty$, then also $V^{r e l}\left(t_{0}, x_{0}\right)=-\infty$. Assume next that $\left(t_{0}, x_{0}\right) \in \operatorname{dom}\left(\bar{V}^{\text {rel }}\right)$ and consider $(\bar{x}(\cdot), \bar{\ell}(\cdot))$ solving the inclusion (15) and satisfying (16). By the Castaing representation theorem there exist measurable selections $u_{i}(t) \in U(t), i \geq 1$ defined on $\mathbb{R}_{+}$such that $U(t)=$ $\overline{\bigcup_{i \geq 1}\left\{u_{i}(t)\right\}}$ for every $t \geq 0$. Consider $u_{0} \in \mathcal{U}$ such that the function defined 
by $k(t):=\left|f\left(t, 0, u_{0}(t)\right)\right|+\left|L\left(t, 0, u_{0}(t)\right)\right|$ is locally integrable on $\mathbb{R}_{+}$. Let the sets $U_{j}(t)$ be defined in the same way as in the proof of Theorem 5.3 for $g=(f, L)$.

Then for a.e. $t \geq t_{0}$

$$
\left(\bar{x}^{\prime}(t), \bar{\ell}(t)\right) \in \overline{\bigcup_{j \geq 1} c o\left\{(f(t, \bar{x}(t), u), L(t, \bar{x}(t), u)): u \in U_{j}(t)\right\}} .
$$

Define

$$
\gamma_{j}(t):=d_{c o}\left\{(f(t, \bar{x}(t), u), L(t, \bar{x}(t), u)): u \in U_{j}(t)\right\}\left(\bar{x}^{\prime}(t), \bar{\ell}(t)\right)
$$

and observe that for a.e. $t \geq t_{0}$ we have $\lim _{j \rightarrow \infty} \gamma_{j}(t)=0$.

Fix $\varepsilon>0$. By our assumptions there exists $\tau>T_{0}$ such that for every $j$ and any solution $(x, \ell)$ of the inclusion

$$
\left(x^{\prime}(t), \ell(t)\right) \in \operatorname{co}\left\{(f(t, x(t), u), L(t, x(t), u)): u \in U_{j}(t)\right\} \text { for a.e. } t \geq t_{0}
$$

we have $\int_{\tau}^{\infty}|\ell(t)| d t<\varepsilon / 3$ and $\int_{\tau}^{\infty}|\bar{\ell}(t)| d t<\varepsilon / 3$.

Then, as in the proof of Theorem 5.3 applied with $T=\tau$ and the initial time $t_{0}$ instead of zero, it follows that for every sufficiently large $j$ there exist an absolutely continuous $x_{j}:\left[t_{0}, \tau\right] \rightarrow \mathbb{R}^{n}$ and $\ell_{j} \in L^{1}\left(\left[t_{0}, \tau\right] ; \mathbb{R}\right)$ solving (17) on $\left[t_{0}, \tau\right]$ and satisfying

$$
\left|\int_{t_{0}}^{\tau} \ell_{j}(t) d t-\int_{t_{0}}^{\tau} \bar{\ell}(t) d t\right|<\frac{\varepsilon}{3}
$$

Consider any trajectory-control pair $(x, u)$ of (2) with $t_{0}$ replaced by $\tau$ and $x_{0}$ by $x_{j}(\tau)$. We extend the trajectory-control pair $\left(x_{j}, u_{j}\right)$ on the time interval $(\tau, \infty)$ by the pair $(x, u)$ and set $\ell_{j}(t)=L(t, x(t), u(t))$ for all $t>\tau$. Hence

$$
\bar{V}^{r e l}\left(t_{0}, x_{0}\right)<\int_{t_{0}}^{\tau} \bar{\ell}(t) d t+\frac{\varepsilon}{3}<\int_{t_{0}}^{\tau} \ell_{j}(t) d t+\frac{2 \varepsilon}{3}<\int_{t_{0}}^{\infty} \ell_{j}(t) d t+\varepsilon .
$$

By the measurable selection theorem, any solution of (17) satisfies the relaxed system (13) for some measurable $\left\{\left(u_{i}, \lambda_{i}\right)\right\}_{i=0}^{n}$. Therefore

$$
\bar{V}^{r e l}\left(t_{0}, x_{0}\right) \leq V^{r e l}\left(t_{0}, x_{0}\right)+\varepsilon .
$$

Hence, by the arbitrariness of $\varepsilon>0$, we get $\bar{V}^{r e l}\left(t_{0}, x_{0}\right) \leq V^{r e l}\left(t_{0}, x_{0}\right)$.

It remains to consider the case $\bar{V}^{r e l}\left(t_{0}, x_{0}\right)=+\infty$. Then there exist $\left(\bar{x}^{s}, \bar{\ell}^{s}\right)$ solving (15) such that $\lim _{s \rightarrow \infty} \int_{t_{0}}^{\infty} \bar{\ell}^{s}(t) d t=+\infty$. By the same arguments as above we can find $\left(x^{s}, v^{s}, \Lambda^{s}\right)$ satisfying (13) such that

$$
\lim _{s \rightarrow \infty} \int_{t_{0}}^{\infty} \sum_{i=0}^{n} \lambda_{i}^{s}(t) L\left(t, x^{s}(t), u_{i}^{s}(t)\right) d t=+\infty .
$$

Hence $V\left(t_{0}, x_{0}\right)=+\infty$. This completes the proof. 


\section{Relaxation in the PRESEnce of State CONStraints}

In this section we consider the problem (12), (13), (3).

When $K \neq \mathbb{R}^{n}$, in general, it may happen that $\operatorname{dom}(V)$ is strictly contained in $\operatorname{dom}\left(V^{r e l}\right)$ even under all the assumptions of the previous section. In fact one needs to impose some geometric restrictions on $f$ on the boundary of $K$, the so called Relaxed Inward Pointing Condition, to obtain the relaxation theorem.

We denote by $\left(I P C^{r e l}\right)$ the following assumption:

$$
\left\{\begin{array}{l}
\forall t \in[0, \infty), \forall x \in \operatorname{bd}(K), \\
\forall v \in \operatorname{Limsup}_{(s, y) \rightarrow(t, x)} f(s, y, U(s)) \text { with } \max _{n \in N_{K}^{1}(x)}\langle n, v\rangle \geq 0, \\
\exists w \in \operatorname{Liminf}_{(s, y) \rightarrow(t, x)} \operatorname{cof}(s, y, U(s)) \text { with } \max _{n \in N_{K}^{1}(x)}\langle n, w-v\rangle<0 .
\end{array}\right.
$$

Remark 6.1. If $f$ is continuous and the set-valued map $U(\cdot)$ is continuous and has compact nonempty images, then the above condition takes a simpler form:

$$
\left\{\begin{array}{l}
\forall t \in[0, \infty), \forall x \in \mathrm{bd}(K), \forall u \in U(t) \text { with } \max _{n \in N_{K}^{1}(x)}\langle n, f(t, x, u)\rangle \geq 0 \\
\exists w \in \operatorname{cof}(t, x, U(t)) \text { with } \max _{n \in N_{K}^{1}(x)}\langle n, w-f(t, x, u)\rangle<0
\end{array}\right.
$$

Theorem 6.2. Under all the assumptions of Theorem 5.1 with (H1) $i$ ) replaced by (6), suppose $\left(I P C^{\text {rel }}\right)$ and that $f, L$ are locally bounded on $\mathbb{R}_{+} \times$ $\operatorname{bd}(K)$.

Then $\operatorname{dom}\left(V^{\text {rel }}\right)=\mathbb{R}_{+} \times K, V^{\text {rel }}=V$ and for every $\left(t_{0}, x_{0}\right) \in \mathbb{R}_{+} \times K$, there exists $\left(\bar{x}(\cdot), \bar{v}(\cdot)=\left(\bar{u}_{0}(\cdot), \ldots, \bar{u}_{n}(\cdot)\right), \bar{\Lambda}(\cdot)=\left(\bar{\lambda}_{0}(\cdot), \ldots, \bar{\lambda}_{n}(\cdot)\right)\right)$ satisfying (13) such that $\bar{x}(t) \in K$ for every $t \geq t_{0}$ and

$$
V^{r e l}\left(t_{0}, x_{0}\right)=\int_{t_{0}}^{\infty} \hat{L}(t, \bar{x}(t), \bar{v}(t), \bar{\Lambda}(t)) d t .
$$

Remark 6.3. We replaced (H1) i) by (6) just to fit the assumptions of [26]. However in [26] assumption (6) is needed only to get uniform bounds on trajectories of a differential inclusion on a finite time interval. By what precedes we know that such bounds follow also from (H1) i). Hence the above result is valid as well with assumption (H1) i) instead of (6).

Proof. From [26, Theorem 3.3] applied with $\alpha=0, \beta=0$ and finite time intervals $\left[t_{0}+k, t_{0}+k+1\right]$ instead of $[0,1]$, we deduce, using the induction argument, that for every $\left(t_{0}, x_{0}\right) \in \mathbb{R}_{+} \times K$, there exists a feasible solution of $(13)$ defined on $\left[t_{0}, \infty\right)$. Hence, in the same way as before, $\operatorname{dom}\left(V^{r e l}\right)=$ $\mathbb{R}_{+} \times K$. Since $\hat{f}, \hat{L}, \widehat{U}$ satisfy $(\mathrm{H} 1)$ the third statement follows from Theorem 4.1. To prove the second one, observe that $V^{r e l} \geq V$. Let $\left(t_{0}, x_{0}\right) \in \mathbb{R}_{+} \times K$ and $(\bar{x}(\cdot), \bar{v}(\cdot), \bar{\Lambda}(\cdot))$ be optimal for the relaxed problem. Fix any $\varepsilon>0$ and $T>t_{0}$ such that $\int_{T}^{\infty}(\alpha(s)+\delta(s)) d s \leq \varepsilon / 2$. Then

$$
V^{r e l}\left(t_{0}, x_{0}\right) \leq \frac{\varepsilon}{2}+\int_{t_{0}}^{T} \hat{L}(t, \bar{x}(t), \bar{v}(t), \bar{\Lambda}(t)) d t
$$


From [26, Corollary 3.4] we deduce that there exists a trajectory-control pair $\left(x_{\varepsilon}, u_{\varepsilon}\right)$ of $(2),(3)$ defined on $\left[t_{0}, T\right]$ such that

$$
\left|\int_{t_{0}}^{T} \hat{L}(t, \bar{x}(t), \bar{v}(t), \bar{\Lambda}(t)) d t-\int_{t_{0}}^{T} L\left(t, x_{\varepsilon}(t), u_{\varepsilon}(t)\right) d t\right|<\frac{\varepsilon}{2} .
$$

Applying [26, Theorem 3.3 and Corollary 3.4] and an induction argument, we extend the feasible trajectory-control pair $\left(x_{\varepsilon}, u_{\varepsilon}\right)$ on the time interval $[T, \infty)$.

The proof ends in the same way as the one of Theorem 5.1.

To investigate locally Lipschitz solutions of the Hamilton-Jacobi equation we need to recall the result below proved in [10].

Consider the infinite horizon optimal control problem $\mathcal{B}_{\infty}$ :

$$
\operatorname{maximize} \int_{t_{0}}^{\infty} e^{-\lambda t} l(t, x(t), u(t)) d t
$$

over all feasible trajectory-control pairs $(x(\cdot), u(\cdot))$ of $(2)$ defined on $\left[t_{0}, \infty\right)$, where $\lambda>0, f, U, K$ are as in the introduction and $l: \mathbb{R}_{+} \times \mathbb{R}^{n} \times \mathbb{R}^{m} \rightarrow \mathbb{R}$ is a given function. Let $V_{\lambda}$ be the corresponding value function and $V_{\lambda}^{r e l}$ be the value function of the relaxed problem (12), (13) under sate constraint (3) for $L(t, x, u)=e^{-\lambda t} l(t, x, u)$.

We denote by (H2) the following assumptions on $f$ and $l$ :

(i) there exists $q \in \mathcal{L}_{\text {loc }}$ such that for a.e. $t \in \mathbb{R}_{+}$,

$$
\sup _{u \in U(t)}(|f(t, x, u)|+|l(t, x, u)|) \leqslant q(t), \quad \forall x \in \operatorname{bd}(K) ;
$$

(ii) for all $(t, x) \in \mathbb{R}_{+} \times \mathbb{R}^{n}$ the set $\{(f(t, x, u), l(t, x, u)): u \in U(t)\}$ is compact;

(iii) $l$ is bounded and there exist $c \in L_{\text {loc }}^{1}\left(\mathbb{R}_{+} ; \mathbb{R}_{+}\right)$and $k \in \mathcal{L}_{\text {loc }}$ such that for a.e. $t \in \mathbb{R}_{+}$and for all $x, y \in \mathbb{R}^{n}$ and $u \in U(t)$,

$$
|f(t, x, u)-f(t, y, u)|+|l(t, x, u)-l(t, y, u)| \leqslant k(t)|x-y|,|f(t, 0, u)| \leqslant c(t)
$$

(iv) $\limsup _{t \rightarrow \infty} \frac{1}{t} \int_{0}^{t}(c(s)+k(s)) d s<\infty$;

(v) the mappings $f, l$ are Carathéodory;

We also need the following (stronger than before) Inward Pointing Condition linking the dynamics to the state constraints : $\left(I P C^{\infty}\right)$

$$
\left\{\begin{array}{l}
\exists \eta>0, \rho>0, M \geqslant 0 \text { such that for a.e. } t \in \mathbb{R}_{+}, \forall y \in \operatorname{bd}(K)+\eta B, \\
\forall u \in U(t) \text { with } \sup _{n \in N_{y, \eta}^{1}}\langle n, f(t, y, u)\rangle \geqslant 0, \\
\exists w \in\left\{w^{\prime} \in U(t):\left|f\left(t, y, w^{\prime}\right)-f(t, y, u)\right| \leqslant M\right\} \text { such that } \\
\sup _{n \in N_{y, \eta}^{1}}\{\langle n, f(t, y, w)\rangle,\langle n, f(t, y, w)-f(t, y, u)\rangle\} \leqslant-\rho,
\end{array}\right.
$$

where $N_{y, \eta}^{1}:=\left\{n \in N_{K}^{1}(x): x \in \operatorname{bd}(K) \cap B(y, \eta)\right\}$. 
Theorem 6.4. Assume $(\mathrm{H} 2)$ and $\left(\mathrm{IPC}^{\infty}\right)$. Then there exist $C>1$ and $\kappa>0$ such that for every $\lambda>\kappa$ and every $t \geqslant 0$ the function $V_{\lambda}(t, \cdot)$ is $C e^{-(\lambda-\kappa) t}$ -Lipschitz continuous on $K$.

Furthermore, if $f$ is also bounded, then for every $\lambda>\kappa, V_{\lambda}$ is locally Lipschitz on $\mathbb{R}_{+} \times K$.

Remark 6.5. In [10] the constants $C$ and $\kappa$ are obtained explicitly. They depend solely on $\rho, M, q, c, k$.

Theorem 6.6. Assume $(\mathrm{H} 2)$ and $\left(\mathrm{IPC}^{\infty}\right)$. Then, for all large $\lambda>0, V_{\lambda}=$ $V_{\lambda}^{\text {rel on }} \mathbb{R}_{+} \times K$.

Proof of Theorem 6.4 is lengthly and highly technical. The presence of the discount factor plays an important role there. The main idea is to use the so called Neighboring Feasible Trajectory (NFT) theorem from [26]. Theorem 6.6 is proved thanks to the continuity of $V_{\lambda}(t, \cdot)$ and the technique developed in [14].

\section{Relation to a finite horizon Bolza problem}

Let $t_{0} \geq 0$ and $T>t_{0}$. Define $g_{T}(x)=V(T, x)$ for all $x \in K, g_{T}(x)=-\infty$ for all $x \notin K$ and consider the Bolza problem $\mathcal{B}_{T}$

$$
\operatorname{maximize}\left(g_{T}(x(T))+\int_{t_{0}}^{T} L(t, x(t), u(t)) d t\right)
$$

over all trajectory-control pairs $(x, u)$ of the system

$$
\begin{cases}x^{\prime}(t)=f(t, x(t), u(t)), & u(t) \in U(t) \text { for a.e. } t \in\left[t_{0}, T\right] \\ x\left(t_{0}\right)=x_{0}, & x(t) \in K \forall t \in\left[t_{0}, T\right] .\end{cases}
$$

The value function $V^{B}$ of the Bolza problem $\mathcal{B}_{T}$ is given by : for every $\left(s_{0}, y_{0}\right) \in\left[t_{0}, T\right] \times K$

$$
V^{B}\left(s_{0}, y_{0}\right)=\sup \left(g_{T}(x(T))+\int_{s_{0}}^{T} L(t, x(t), u(t)) d t\right)
$$

over all feasible trajectory-control pairs $(x, u)$ of $(5)$ defined on $\left[s_{0}, T\right]$ with $x\left(s_{0}\right)=y_{0}$. Again, if there is no feasible trajectory-control pair of (5) defined on $\left[s_{0}, T\right]$ and satisfying $x\left(s_{0}\right)=y_{0}$, then we set $V^{B}\left(s_{0}, y_{0}\right)=-\infty$.

If (H1) holds true, then, by Theorem $4.1, V(T, \cdot)$ is upper semicontinuous and, by the well known existence theorems for finite horizon problems, for every $y_{0} \in \operatorname{dom}\left(V^{B}\left(s_{0}, \cdot\right)\right)$, the above Bolza problem has an optimal solution.

Proposition 7.1. Assume (H1) i) - v). Then $V^{B}\left(s_{0}, y_{0}\right)=V\left(s_{0}, y_{0}\right)$ for every $\left(s_{0}, y_{0}\right) \in\left[t_{0}, T\right] \in K$. Moreover, if $(\bar{x}, \bar{u})$ is optimal for the infinite horizon problem at $\left(t_{0}, x_{0}\right)$, then the restriction of $(\bar{x}, \bar{u})$ to $\left[t_{0}, T\right]$ is optimal for $\mathcal{B}_{T}$. 
Proof. By (7) and (H1) iv), for any $R>0$ there exists $M>0$ such that for any trajectory-control pair $(x, u)$ of $(5)$ defined on $\left[s_{0}, T\right]$ with $T \geq$ $s_{0} \geq 0$, satisfying $x\left(s_{0}\right)=y_{0}$ and $T+\left|y_{0}\right| \leq R$ we have $|x(T)| \leq M$ and $\int_{s_{0}}^{T}|L(t, x(t), u(t))| d t \leq M$. As in the proof of Theorem 4.1 it can be shown that $V^{B}$ and $V$ take values in $[-\infty, \infty)$.

Let $\left(s_{0}, y_{0}\right) \in\left[t_{0}, T\right] \times K$. If $V^{B}\left(s_{0}, y_{0}\right)=-\infty$, then $V^{B}\left(s_{0}, y_{0}\right) \leq V\left(s_{0}, y_{0}\right)$. If it is finite, then fix $\varepsilon>0$ and consider a feasible trajectory-control pair $\left(x_{\varepsilon}, u_{\varepsilon}\right)$ with $x_{\varepsilon}\left(s_{0}\right)=y_{0}$ defined on $\left[s_{0}, T\right]$ such that

$$
V^{B}\left(s_{0}, y_{0}\right) \leq g_{T}\left(x_{\varepsilon}(T)\right)+\int_{s_{0}}^{T} L\left(s, x_{\varepsilon}(s), u_{\varepsilon}(s)\right) d s+\frac{\varepsilon}{2} .
$$

Since $g_{T}\left(x_{\varepsilon}(T)\right)$ is finite, we deduce that $\left(T, x_{\varepsilon}(T)\right) \in \operatorname{dom}(V)$. Consider a feasible trajectory-control pair $(x, u)$ of $(5)$ on $[T, \infty)$ such that $x(T)=$ $x_{\varepsilon}(T)$ and $V\left(T, x_{\varepsilon}(T)\right) \leq \int_{T}^{\infty} L(s, x(s), u(s)) d s+\varepsilon / 2$. Set $\left(x_{\varepsilon}(s), u_{\varepsilon}(s)\right)=$ $(x(s), u(s)))$ for $s>T$. Then,

$$
\begin{aligned}
V^{B}\left(s_{0}, y_{0}\right) & \leq \int_{s_{0}}^{T} L\left(s, x_{\varepsilon}(s), u_{\varepsilon}(s)\right) d s+\int_{T}^{\infty} L\left(s, x_{\varepsilon}(s), u_{\varepsilon}(s)\right) d s+\varepsilon \\
& \leq V\left(s_{0}, y_{0}\right)+\varepsilon .
\end{aligned}
$$

Since $\varepsilon>0$ is arbitrary, $V^{B}\left(s_{0}, y_{0}\right) \leq V\left(s_{0}, y_{0}\right)$. On the other hand, if $V\left(s_{0}, y_{0}\right)=-\infty$, then $V\left(s_{0}, y_{0}\right) \leq V^{B}\left(s_{0}, y_{0}\right)$. If it is finite, then consider a feasible trajectory-control pair $\left(x_{\varepsilon}, u_{\varepsilon}\right)$ of $(5)$ on $\left[s_{0}, \infty\right)$ satisfying $x_{\varepsilon}\left(s_{0}\right)=y_{0}$ and such that $V\left(s_{0}, y_{0}\right) \leq \int_{s_{0}}^{\infty} L\left(s, x_{\varepsilon}(s), u_{\varepsilon}(s)\right) d s+\varepsilon$. Then

$$
\begin{aligned}
& V\left(s_{0}, y_{0}\right) \leq \int_{s_{0}}^{T} L\left(s, x_{\varepsilon}(s), u_{\varepsilon}(s)\right) d s+\int_{T}^{\infty} L\left(s, x_{\varepsilon}(s), u_{\varepsilon}(s)\right) d s+\varepsilon \\
& \leq \int_{s_{0}}^{T} L\left(s, x_{\varepsilon}(s), u_{\varepsilon}(s)\right) d s+V\left(T, x_{\varepsilon}(T)\right)+\varepsilon \leq V^{B}\left(s_{0}, y_{0}\right)+\varepsilon .
\end{aligned}
$$

By the arbitrariness of $\varepsilon>0$, this yields $V\left(s_{0}, y_{0}\right) \leq V^{B}\left(s_{0}, y_{0}\right)$. Hence $V\left(s_{0}, y_{0}\right)=V^{B}\left(s_{0}, y_{0}\right)$. The point $\left(s_{0}, y_{0}\right) \in\left[t_{0}, T\right] \times K$ being arbitrary, we deduce the first statement. The second one is a simple consequence of the dynamic programming principle.

\section{MAXIMUM PRINCIPLE AND SENSITIVITY RELATION}

When state constraints are present, then, in general, the familiar maximum principle does not hold unless the optimal trajectory takes values in the interior of $K$ only. Furthermore, for problems under state constraints the adjoint state may be discontinuous, even for finite horizon problems.

In this section we prove the maximum principle using the Hamiltonian $H$ defined in the introduction. Recall that $H(t, x, \cdot)$ is convex and if $f(t, \cdot, u), L(t, \cdot, u)$ are locally Lipschitz uniformly in $u \in U(t)$, then $H(t, \cdot, \cdot)$ is locally Lipschitz. Then we denote by $\partial H(t ; y, q)$ the generalized gradient of $H(t, \cdot, \cdot)$ at $(y, q)$. It has convex compact images and the set-valued map $(y, q) \leadsto \partial H(t ; y, q)$ is upper semicontinuous. 
If $V$ is locally Lipschitz on $\mathbb{R}_{+} \times K$, then define for $(t, x) \in \mathbb{R}_{+} \times K$,

$$
\partial^{i n t} V(t, x):=\text { coLimsup } \underset{\substack{(s, y) \rightarrow(t, x) \\ y \in \operatorname{int}(K)}}{ }\{\nabla V(s, y)\} .
$$

Observe that the set $\partial^{i n t} V(t, x)$ is compact. It coincides with the generalized gradient of $V$ at $(t, x)$ whenever $x \in \operatorname{int}(K)$.

Below we denote by $\partial_{x}^{L,-} V\left(t_{0}, x_{0}\right)$ the limiting subdifferential of $V\left(t_{0}, \cdot\right)$ at $x_{0}$. Recalling the notation introduced in Section 4,

$$
G(t, x):=\{(f(t, x, u), L(t, x, u)): u \in U(t)\},
$$

we state the maximum principle under state constraints.

Theorem 8.1. Assume (IPC $\left.{ }^{r e l}\right)$, (H1) ii)-v) with $\omega_{R}(t, r)=\bar{c}_{R}(t) r$, where for all $R>0, \bar{c}_{R} \in L_{\text {loc }}^{1}\left(\mathbb{R}_{+} ; \mathbb{R}_{+}\right)$, that $G(t, x)$ is compact for every $(t, x) \in$ $\mathbb{R}_{+} \times \mathbb{R}^{n}$ and that there exists $c>0$ such that for a.e. $t \geq 0$,

$$
\sup _{u \in U(t)}(|f(t, x, u)|+|L(t, x, u)|) \leq c(|x|+1) \forall x \in \mathbb{R}^{n} .
$$

Then

I) if for all large $j \in \mathbb{N}$, the function $V(j, \cdot)$ is locally Lipschitz on $K$, then $V$ is locally Lipschitz on $\mathbb{R}_{+} \times K$.

II) if $(\bar{x}, \bar{u})$ is optimal for the infinite horizon problem at $\left(t_{0}, x_{0}\right) \in \mathbb{R}_{+} \times$ $\operatorname{int}(K)$, then there exist $p \in W_{\text {loc }}^{1,1}\left(t_{0}, \infty ; \mathbb{R}^{n}\right)$, positive Borel measure $\mu$ on $\left[t_{0}, \infty\right)$, and a Borel measurable selection $\nu(t) \in N_{K}(\bar{x}(t)) \cap B$ defined on $\left[t_{0}, \infty\right)$ such that for $q(t):=p(t)+\eta(t)$ with

$$
\eta(t):= \begin{cases}\int_{\left[t_{0}, t\right]} \nu(s) d \mu(s) & t \in\left(t_{0}, \infty\right) \\ 0 & t=t_{0},\end{cases}
$$

the following relations are satisfied:

(i) the Hamiltonian inclusion

$$
\left(-p^{\prime}(t), \bar{x}^{\prime}(t)\right) \in \partial H(t ; \bar{x}(t), q(t)) \text { for a.e. } t \in\left[t_{0}, \infty\right) ;
$$

(ii) the maximality condition

$$
\left\langle q(t), \bar{x}^{\prime}(t)\right\rangle+L(t, \bar{x}(t), \bar{u}(t))=H(t, \bar{x}(t), q(t)) \text { for a.e. } t \in\left[t_{0}, \infty\right) ;
$$

(iii) the sensitivity relation

$$
(-H(t, \bar{x}(t), q(t)), q(t)) \in \partial^{i n t} V(t, \bar{x}(t)) \text { for a.e. } t \in\left[t_{0}, \infty\right) ;
$$

(iv) the transversality condition at the initial time

$$
p\left(t_{0}\right) \in \partial_{x}^{L,-} V\left(t_{0}, x_{0}\right) .
$$

Furthermore, if $H$ is continuous, then

$$
(-H(t, \bar{x}(t), q(t)), q(t)) \in \partial^{i n t} V(t, \bar{x}(t)) \forall t \in\left(t_{0}, \infty\right) .
$$




\section{Remark 8.2.}

a) Recall that Theorem 6.4 provides sufficient conditions for the local Lipschitz continuity of $V$ on $\mathbb{R}_{+} \times K$. Instead one can also assume that (H2) holds true for $t \in[j, \infty)$ and some integer $j$ (in place of $[0, \infty)$ ) to deduce Lipschitz continuity of $V$ on $[j, \infty) \times K$.

b) It is not difficult to realize that $(i)$ contains the maximality condition (ii). We have stated $(i i)$ in order to underline the link with the more familiar maximum principle.

Proof. For every sufficiently large integer $j>t_{0}$, let $g_{j}: \mathbb{R}^{n} \rightarrow \mathbb{R}$ be a locally Lipschitz function that coincides with $V(j, \cdot)$ on $K$. Consider the Mayer problem $\left(\mathcal{M}_{j}\right)$

$$
V^{j}\left(t_{0}, x_{0}, z_{0}\right)=\sup \left(g_{j}(x(j))+z(j)\right)
$$

over all the trajectory-control pairs of the control system

$$
\begin{cases}x^{\prime}(t)=f(t, x(t), u(t)) & \text { a.e. } t \in\left[t_{0}, j\right] \\ z^{\prime}(t)=L(t, x(t), u(t)) & \text { a.e. } t \in\left[t_{0}, j\right] \\ x\left(t_{0}\right)=x_{0}, z\left(t_{0}\right)=z_{0} & \\ u(t) \in U(t) & \text { a.e. } t \in\left[t_{0}, j\right] \\ x(t) \in K & \forall t \in\left[t_{0}, j\right] .\end{cases}
$$

From [26, Theorem 5.1] (after rewriting the above Mayer problem as a minimization problem), we deduce that $V^{j}$ is locally Lipschitz on $[0, j] \times$ $K \times \mathbb{R}$ whenever $j$ is sufficiently large. It is not difficult to realize that $V^{j}\left(t_{0}, x_{0}, 0\right)=V^{B}\left(t_{0}, x_{0}\right)$ for every $\left(t_{0}, x_{0}\right) \in[0, j] \times K$, where $V^{B}$ is the value function of the Bolza problem (introduced in the previous section) for $T=j$. Hence, by Proposition 7.1, $V$ is locally Lipschitz continuous on $\mathbb{R}_{+} \times K$.

To prove the second statement of our theorem, consider an optimal pair $(\bar{x}, \bar{u})$ of the infinite horizon problem at $\left(t_{0}, x_{0}\right) \in \mathbb{R}_{+} \times \operatorname{int}(K)$. Setting

$$
\bar{z}(t)=\int_{t_{0}}^{t} L(s, \bar{x}(s), \bar{u}(s)) d s
$$

it follows that the restriction of $(\bar{x}, \bar{z}, \bar{u})$ to $\left[t_{0}, j\right]$ is optimal for $\left(\mathcal{M}_{j}\right)$ at $\left(t_{0}, x_{0}, 0\right)$. By [26, Theorem 5.3] (after rewriting the above Mayer problem as a minimization problem on the interval $\left[t_{0}, j\right]$ instead of $\left.[0,1]\right)$, for every integer $j>t_{0}$ there exist an absolutely continuous function $p_{j}$, a positive Borel measure $\mu_{j}$ and a Borel measurable selection $\nu_{j}(s) \in N_{K}(\bar{x}(s)) \cap B$ $\mu_{j}$ - a.e. defined on the time interval $\left[t_{0}, j\right]$ such that, setting

$$
\eta_{j}(t):= \begin{cases}\int_{\left[t_{0}, t\right]} \nu_{j}(s) d \mu_{j}(s) & t \in\left(t_{0}, j\right] \\ 0 & t=t_{0}\end{cases}
$$

and $q_{j}(t):=p_{j}(t)+\eta_{j}(t)$, the following relations hold true for a.e. $t \in\left[t_{0}, j\right]$ :

$$
\begin{gathered}
\left(-p_{j}^{\prime}(t), \bar{x}^{\prime}(t)\right) \in \partial H\left(t ; \bar{x}(t), q_{j}(t)\right) ; \\
\left\langle q_{j}(t), f(t, \bar{x}(t), \bar{u}(t))\right\rangle+L(t, \bar{x}(t), \bar{u}(t))=H\left(t, \bar{x}(t), q_{j}(t)\right) ;
\end{gathered}
$$




$$
\begin{gathered}
\left(-H\left(t, \bar{x}(t), q_{j}(t)\right), q_{j}(t)\right) \in \partial^{i n t} V(t, \bar{x}(t)) ; \\
p_{j}\left(t_{0}\right) \in \partial_{x}^{L,-} V\left(t_{0}, x_{0}\right) .
\end{gathered}
$$

Let $k>t_{0}$ be a fixed integer.

Step 1: Since $x_{0} \in \operatorname{int}(K)$ and $V$ is locally Lipschitz, we deduce from (22) that the sequence $\left\{p_{j}\left(t_{0}\right)\right\}_{j}$ is bounded. The measure $\mu_{j}$ being regular, we know that $q_{j}$ is right continuous on $\left(t_{0}, j\right)$. Since $V$ is locally Lipschitz on $\mathbb{R}_{+} \times K$ from (21) we deduce that the functions $\left\{q_{j}\right\}_{j>k}$ are equibounded on $\left[t_{0}, k\right]$.

On the other hand, if $R>0$ is so that $\sup _{t \in\left[t_{0}, k\right]}|\bar{x}(t)|<R$, then for a.e. $t \in$ $\left[t_{0}, k\right]$ and all $(a, b) \in \partial H\left(t ; \bar{x}(t), q_{j}(t)\right) \subset \mathbb{R}^{n} \times \mathbb{R}^{n}$ we have $\left.|a| \leq c_{R}(t) \mid q_{j}(t)\right) \mid+$ $\bar{c}_{R}(t)$. This and (19) imply that $\left.\left|p_{j}^{\prime}(t)\right| \leq c_{R}(t) \mid q_{j}(t)\right) \mid+\bar{c}_{R}(t)$ a.e. in $\left[t_{0}, k\right]$. Hence also the mappings $\left\{p_{j}\right\}_{j}$ are equibounded on $\left[t_{0}, k\right]$ and therefore so are $\left\{\eta_{j}\right\}_{j}$.

For every $j>k$ define the positive measure $\bar{\mu}_{j}$ on Borel subsets of $\left[t_{0}, k\right]$ by $\bar{\mu}_{j}(A)=\int_{A}\left|\nu_{j}(t)\right| d \mu_{j}(t)$ for any Borel set $A \subset\left[t_{0}, k\right]$. We claim that $\bar{\mu}_{j}\left(\left[t_{0}, k\right]\right)$ are equibounded. Indeed, the inward pointing condition implies that for every $t \geq t_{0}$, $\operatorname{int}\left(C_{K}(\bar{x}(t))\right) \neq \emptyset$. Thus the set valued map $t \rightsquigarrow C_{K}(\bar{x}(t))$ is lower semicontinuous on $\left[t_{0}, \infty\right)$. By $[18$, proof of Lemma 11] we know that there exists a continuous selection $\psi(t) \in C_{K}(t)$ defined on $\left[t_{0}, k\right]$ such that

$$
\sup _{n \in N_{K}^{1}(\bar{x}(t))}\langle n, \psi(t)\rangle \leqslant-2 \quad \forall t \in\left[t_{0}, k\right] \text { with } \bar{x}(t) \in \operatorname{bd}(K) .
$$

Consider $\bar{\psi} \in C^{\infty}\left(\left[t_{0}, k\right] ; \mathbb{R}^{n}\right)$ such that $\sup _{t \in\left[t_{0}, k\right]}|\psi(s)-\bar{\psi}(s)| \leqslant 1$. Then

$$
\sup _{n \in N_{K}^{1}(\bar{x}(t))}\langle n, \bar{\psi}(t)\rangle \leqslant-1 \quad \forall t \in\left[t_{0}, k\right] \text { with } \bar{x}(t) \in \operatorname{bd}(K) .
$$

Consequently, for every $j>k$,

$$
\begin{aligned}
& \int_{\left[t_{0}, k\right]}\left\langle\bar{\psi}(s), \nu_{j}(s)\right\rangle d \mu_{j}(s)=\int_{\left[t_{0}, k\right] \cap\left\{s: \nu_{j}(s) \neq 0\right\}}\left\langle\bar{\psi}(s), \frac{\nu_{j}(s)}{\left|\nu_{j}(s)\right|}\right\rangle\left|\nu_{j}(s)\right| d \mu_{j}(s) \\
& \leqslant-\int_{\left[t_{0}, k\right] \cap\left\{s: \nu_{j}(s) \neq 0\right\}}\left|\nu_{j}(s)\right| d \mu_{j}(s)=-\int_{\left[t_{0}, k\right]}\left|\nu_{j}(s)\right| d \mu_{j}(s) .
\end{aligned}
$$

Using that $\left\{\eta_{j}\right\}_{j}$ are equibounded on $\left[t_{0}, k\right]$ and have bounded total variation, integrating by parts we deduce from the above inequality that for some constant $C \geqslant 0$ and all $j>k$,

$$
\begin{aligned}
& \int_{\left[t_{0}, k\right]}\left|\nu_{j}(s)\right| d \mu_{j}(s) \leqslant \int_{\left[t_{0}, k\right]}\left\langle-\bar{\psi}(s), \nu_{j}(s)\right\rangle d \mu_{j}(s)=\int_{\left[t_{0}, k\right]}-\bar{\psi}(s) d \eta_{j}(s) \\
& =-\eta_{j}(k) \bar{\psi}(k)+\int_{\left[t_{0}, k\right]} \eta_{j}(s) \bar{\psi}^{\prime}(s) d s \leqslant C\left(\sup _{s \in\left[t_{0}, k\right]}|\bar{\psi}(s)|+\sup _{s \in\left[t_{0}, k\right]}\left|\bar{\psi}^{\prime}(s)\right|\right) .
\end{aligned}
$$

Since $\bar{\psi}(\cdot)$ does not depend on $j$ our claim follows.

Step 2: By Step 1, the Ascoli-Arzelà theorem and the Dunford-Pettis criterion, taking a subsequence and keeping the same notation, we deduce 
that there exists an absolutely continuous function $p^{k}:\left[t_{0}, k\right] \rightarrow \mathbb{R}^{n}$ such that $p_{j} \rightarrow p^{k}$ uniformly on $\left[t_{0}, k\right]$ and $p_{j}^{\prime} \rightarrow\left(p^{k}\right)^{\prime}$ weakly in $L^{1}\left(\left[t_{0}, k\right] ; \mathbb{R}^{n}\right)$. Since the total variations of $\eta_{j}$ are bounded by $\int_{\left[t_{0}, k\right]}\left|\nu_{j}(s)\right| d \mu_{j}(s)$ and $\eta_{j}(0)=0$, from the Helly's selection theorem, taking a subsequence and keeping the same notation, we conclude that there exists a function of bounded variation $\eta^{k}$ on $\left[t_{0}, k\right]$ such that $\eta_{j} \rightarrow \eta^{k}$ pointwise on $\left[t_{0}, k\right]$ and $\eta^{k}\left(t_{0}\right)=0$. Furthermore, there exists a nonnegative finite measure $\mu^{k}$ on $\left[t_{0}, k\right]$ such that, by further extraction of a subsequence and preserving the same notation, $\bar{\mu}_{j} \rightarrow^{*} \mu^{k}$ (weakly-*) in $C\left(\left[t_{0}, k\right] ; \mathbb{R}\right)^{*}$. Let

$$
\gamma_{j}(t):= \begin{cases}\frac{\nu_{j}(t)}{\left|\nu_{j}(t)\right|} & \nu_{j}(t) \neq 0 \\ 0 & \text { otherwise. }\end{cases}
$$

Since $\gamma_{j}(t) \in N_{K}(\bar{x}(t)) \cap B \bar{\mu}_{j}$-a.e. in $\left[t_{0}, k\right]$ is a Borel measurable selection, applying [45, Proposition 9.2.1], we deduce that, for a subsequence $\left\{j_{i}\right\}_{i}$, there exists a Borel measurable function $\nu^{k}$ such that $\nu^{k}(t) \in N_{K}(\bar{x}(t)) \cap B$ $\mu^{k}$-a.e. in $\left[t_{0}, k\right]$ and for every $\phi \in C\left(\left[t_{0}, k\right] ; \mathbb{R}^{n}\right)$

$$
\int_{\left[t_{0}, k\right]}\left\langle\phi(s), \gamma_{j_{i}}(s)\right\rangle d \bar{\mu}_{j_{i}}(s) \rightarrow \int_{\left[t_{0}, k\right]}\left\langle\phi(s), \nu^{k}(s)\right\rangle d \mu^{k}(s) \text { as } i \rightarrow \infty .
$$

Using that for all $t \in\left(t_{0}, k\right]$,

$$
\eta_{j_{i}}(t)=\int_{\left[t_{0}, t\right]} \nu_{j_{i}}(s) d \mu_{j_{i}}(s)=\int_{\left[t_{0}, t\right]} \gamma_{j_{i}}(s) d \bar{\mu}_{j_{i}}(s),
$$

from (23) and the separation theorem it follows that for every $t \in\left(t_{0}, k\right]$

$$
\eta^{k}(t)=\int_{\left[t_{0}, t\right]} \nu^{k}(s) d \mu^{k}(s)
$$

Recall that $\partial H(t ; \cdot, \cdot)$ is upper semicontinuous and has convex compact images and that $\partial_{x}^{L,-} V\left(0, x_{0}\right), \partial^{\text {int }} V(t, \bar{x}(t))$ are closed sets.

Define $q^{k}=p^{k}+\eta^{k}$. Passing to the limits in (20) and (21) a.e. in $\left[t_{0}, k\right]$ for the subsequence $q_{j_{i}}$ when $i \rightarrow \infty$, we deduce that $(i i)$ and (iii) are satisfied on $\left[t_{0}, k\right]$ with $q$ replaced by $q^{k}$. From (22) we obtain (18) for $p^{k}\left(t_{0}\right)$. Taking the weak limit in (19) for the subsequence $p_{j_{i}}^{\prime}$ when $i \rightarrow \infty$ and using the Mazur lemma and the upper semicontinuity of $\partial H(t ; \cdot, \cdot)$ we get $(i)$ on $\left[t_{0}, k\right]$ with $p$ replaced by $p^{k}$ and $q$ by $q^{k}$.

Step 3: Consider now the interval $\left[t_{0}, k+1\right]$. By the same argument as in the second step, taking suitable subsequences $\left\{p_{j_{i_{l}}}\right\}_{l} \subset\left\{p_{j_{i}}\right\}_{i}$ and $\left\{\eta_{j_{i_{l}}}\right\}_{l} \subset$ $\left\{\eta_{j_{i}}\right\}_{i}$, we deduce that there exist an absolutely continuous function $p^{k+1}$, a function of bounded variation $\eta^{k+1}$, a Borel measurable selection $\nu^{k+1}(t) \epsilon$ $N_{K}(\bar{x}(t)) \cap B$ on $\left[t_{0}, k+1\right]$ and a positive Borel measure $\mu^{k+1}$ on $\left[t_{0}, k+1\right]$ which satisfy $(i),(i i),(i i i),(i v)$ on $\left[t_{0}, k+1\right]$ with $p, q$ replaced by $p^{k+1}, q^{k+1}$ 
and moreover, when $\ell \rightarrow \infty$

$$
\begin{aligned}
& p_{j_{i_{l}}} \rightarrow p^{k+1} \text { uniformly on }\left[t_{0}, k+1\right], \\
& p_{j_{i_{l}}}^{\prime} \rightarrow\left(p^{k+1}\right)^{\prime} \text { weakly in } L^{1}\left(\left[t_{0}, k+1\right] ; \mathbb{R}^{n}\right) \\
& q_{j_{i_{l}}} \rightarrow q^{k+1} \text { pointwise on }\left[t_{0}, k+1\right], \\
& \mu_{j_{i_{l}}} \rightarrow^{*} \mu^{k+1} \text { weakly-* in } C\left(\left[t_{0}, k+1\right] ; \mathbb{R}\right)^{*} \\
& \left.p^{k+1}\right|_{\left[t_{0}, k\right]}=p^{k},\left.q^{k+1}\right|_{\left[t_{0}, k\right]}=q^{k},
\end{aligned}
$$

and for all $t \in\left[t_{0}, k+1\right]$

$$
\eta_{j_{i_{l}}}(t) \rightarrow \eta^{k+1}(t)= \begin{cases}\int_{\left[t_{0}, t\right]} \nu^{k+1}(s) d \mu^{k+1}(s) & t \in\left(t_{0}, k+1\right] \\ 0 & t=t_{0}\end{cases}
$$

Furthermore, since $\left.\eta^{k+1}\right|_{\left[t_{0}, k\right]}=\eta^{k}$ and $\left.\mu^{k+1}\right|_{\left[t_{0}, k\right]}=\mu^{k}$, we have

$$
\left.\nu^{k+1}\right|_{\left[t_{0}, k\right]}=\nu^{k} \quad \mu^{k} \text {-a.e. on }\left[t_{0}, k\right] .
$$

The mappings $p^{k+1}, \eta^{k+1}$, and $\nu^{k+1}$ extend $p^{k}, \eta^{k}$, and $\nu^{k}$ respectively on the time interval $\left[t_{0}, k+1\right]$, and the measure $\mu^{k+1}$ extends the measure $\mu^{k}$.

Applying the induction argument we get $p, \eta, \nu$ and $\mu$ defined on $\left[t_{0}, \infty\right)$ and satisfying the second claim of our theorem.

If $H$ is continuous, then the right continuity of $q$ on $\left(t_{0}, \infty\right)$ and the upper semicontinuity of $\partial^{\text {int } V}$ on $\mathbb{R}_{+} \times K$ imply the last statement of our theorem.

\section{HAMilton-JaCOBI EQUATION}

The Hamilton-Jacobi (HJ) equation associated to the non-autonomous infinite horizon optimal control problem is as follows

$$
\partial_{t} W+H\left(t, x, \partial_{x} W\right)=0 \quad \text { on }(0, \infty) \times K,
$$

where $\partial_{t}, \partial_{x}$ denote the partial derivatives of $W$ with respect to $t$ and $x$.

If the value function is differentiable, then it is well known that it satisfies (HJ). If in addition $f$ is time independent and $L(t, x, u)=e^{-\lambda t} \ell(x, u)$ for some $\ell: \mathbb{R}^{n} \times \mathbb{R}^{m} \rightarrow \mathbb{R}$, then $V(t, x)=e^{-\lambda t} V(0, x)$. Setting $W_{1}(x):=V(0, x)$ we deduce from (HJ) that $W_{1}$ is a solution of the following, more familiar in the context of infinite horizon problems, stationary Hamilton-Jacobi equation

$$
-\lambda W+\mathcal{H}\left(x, \partial_{x} W\right)=0 \quad \text { on } K,
$$

where $\mathcal{H}(x, p):=\sup _{u \in U(t)}(\langle p, f(x, u)\rangle+\ell(x, u))$.

Conversely, if $W_{1}$ is a smooth solution of this stationary Hamilton-Jacobi equation, then $W(t, x):=e^{-\lambda t} W_{1}(x)$ solves (HJ). If $f$ and/or $\ell$ are time dependent, then, in general, (HJ) can not be replaced by the above stationary equation.

It is well known that (HJ) may not have differentiable solutions. Furthermore, if a smooth solution does exist, it may be not uniquely defined 
because, in the difference with the finite horizon case, there is no "terminal" condition involved, as in (4). In Section 4 we have shown that under some integrable boundedness assumptions for large times, the value function $V(t, \cdot)$ restricted to its domain of definition converges to zero as $t \rightarrow \infty$. In such a setting, the natural "terminal" condition should be as follows

$$
\lim _{t \rightarrow \infty} \sup _{y \in \operatorname{dom}(W(t, \cdot))}|W(t, y)|=0 .
$$

The value function $V$ being not differentiable, and even possibly discontinuous, solutions of (HJ) have to be understood in a generalized sense. In [11] we have investigated uniqueness of generalized solutions in the class of upper semicontinuous functions satisfying the above "terminal" condition. In this section we restrict our attention to locally Lipschitz continuous solutions on $\mathbb{R}_{+} \times K$ only, the case, where results are much simpler to state and to prove. Then the above "terminal" condition becomes

$$
\lim _{t \rightarrow \infty} \sup _{y \in K}|W(t, y)|=0 .
$$

Denote by (H3) the following assumptions on $f, L$ :

(i) $\exists c \in L_{\text {loc }}^{1}\left(\mathbb{R}_{+} ; \mathbb{R}_{+}\right)$such that for a.e. $t \geqslant 0$ and for all $x \in \mathbb{R}^{n}, u \in U(t)$

$$
|f(t, x, u)|+|L(t, x, u)| \leqslant c(t)(1+|x|) ;
$$

(ii) $\exists k \in \mathcal{L}_{\text {loc }}$ such that for a.e. $t \geqslant 0$ and for all $x, y \in \mathbb{R}^{n}, u \in U(t)$

$$
|f(t, x, u)-f(t, y, u)|+|L(t, x, u)-L(t, y, u)| \leqslant k(t)|x-y|
$$

(iii) $\lim \sup _{t \rightarrow \infty} \frac{1}{t} \int_{0}^{t}(c(s)+k(s)) d s<\infty$;

(iv) $\exists q \in \mathcal{L}_{\text {loc }}$ such that for a.e. $t \geqslant 0$

$$
\sup _{u \in U(t)}(|f(t, x, u)|+|L(t, x, u)|) \leqslant q(t), \forall x \in \operatorname{bd}(K) ;
$$

(v) for a.e. $t \geqslant 0$ and all $x \in \mathbb{R}^{n}, G(t, x)$ is compact;

(vi) There exists $S>0$ such that $|L(t, x, u)| \leq \alpha(t)$ for a.e. $t \geq S$ and all $x \in K, u \in U(t)$, where $\alpha:[S,+\infty) \rightarrow \mathbb{R}_{+}$is integrable on $[S,+\infty)$.

The Outward Pointing Condition $\left(O P C^{\infty}\right)$ is the same as $\left(I P C^{\infty}\right)$ but with $f$ replaced by $-f$.

Theorem 9.1. Assume (H1) iii), vi) and (H3). Let $W: \mathbb{R}_{+} \times K \rightarrow \mathbb{R}$ be a locally Lipschitz function satisfying (24).

1. If $\left(O P C^{\infty}\right)$ holds true, then $W=V$ if and only if $W$ is a bilateral solution of (HJ), that is for a.e. $t>0$

$$
\left\{\begin{array}{l}
p_{t}+H\left(t, x, p_{x}\right)=0 \forall\left(p_{t}, p_{x}\right) \in \partial_{+} W(t, x), \forall x \in \operatorname{int}(K) \\
p_{t}+H\left(t, x, p_{x}\right) \geq 0 \forall\left(p_{t}, p_{x}\right) \in \partial_{+} W(t, x), \forall x \in \operatorname{bd}(K) .
\end{array}\right.
$$


2. If $\left(I P C^{\infty}\right)$ holds true, then $W=V$ if and only if $W$ is a viscosity solution of (HJ), that is for a.e. $t>0$

$$
\left\{\begin{array}{l}
p_{t}+H\left(t, x, p_{x}\right) \leqslant 0 \forall\left(p_{t}, p_{x}\right) \in \partial_{-} W(t, x), \forall x \in \operatorname{int}(K), \\
p_{t}+H\left(t, x, p_{x}\right) \geqslant 0 \forall\left(p_{t}, p_{x}\right) \in \partial_{+} W(t, x), \forall x \in K .
\end{array}\right.
$$

Now, let $l: \mathbb{R}_{+} \times \mathbb{R}^{n} \times \mathbb{R}^{m} \rightarrow \mathbb{R}_{+}, \lambda>0$, and

$$
L(t, x, u)=e^{-\lambda t} l(t, x, u) .
$$

From Theorems 6.2, 9.1 and 6.4 we immediately get the following corollaries.

Corollary 9.2. Assume (27), (H2), (IPC $\left.{ }^{\infty}\right),\left(O P C^{\infty}\right)$ and that $f$ is bounded. Then for every $\lambda>0$ sufficiently large, the value function $V_{\lambda}$ is the only locally Lipschitz function $W: \mathbb{R}_{+} \times K \rightarrow \mathbb{R}$ satisfying for a.e. $t>0$

$$
\left\{\begin{array}{l}
p_{t}+H\left(t, x, p_{x}\right)=0 \forall\left(p_{t}, p_{x}\right) \in \partial_{+} W(t, x), \forall x \in \operatorname{int}(K), \\
p_{t}+H\left(t, x, p_{x}\right) \geqslant 0 \forall\left(p_{t}, p_{x}\right) \in \partial_{+} W(t, x), \forall x \in \operatorname{bd}(K) \\
\lim _{t \rightarrow \infty} \sup _{y \in K}|W(t, y)|=0 .
\end{array}\right.
$$

Corollary 9.3. Assume (27), (H2), (IPC $\left.{ }^{\infty}\right)$ and that $f$ is bounded. Then for every $\lambda>0$ sufficiently large, the value function $V_{\lambda}$ is the only locally Lipschitz function $W: \mathbb{R}_{+} \times K \rightarrow \mathbb{R}$ satisfying for a.e. $t>0$

$$
\left\{\begin{array}{l}
p_{t}+H\left(t, x, p_{x}\right) \leqslant 0 \forall\left(p_{t}, p_{x}\right) \in \partial_{-} W(t, x), \forall x \in \operatorname{int}(K) \\
p_{t}+H\left(t, x, p_{x}\right) \geqslant 0 \forall\left(p_{t}, p_{x}\right) \in \partial_{+} W(t, x), \forall x \in K \\
\lim _{t \rightarrow \infty} \sup _{y \in K}|W(t, y)|=0
\end{array}\right.
$$

The proof of Theorem 9.1 follows the path initiated in [23] that was subsequently applied in many papers, see for instance [27, 29, 30, 31, 25].

We first state several auxiliary results.

Proposition 9.4. The following relations hold true:

- for any $x \in \operatorname{dom}(V(0, \cdot))$, $\limsup _{s \rightarrow 0+, y \rightarrow_{K} x} V(s, y)=V(0, x)$;

- for a.e. $t>0, \forall x \in \operatorname{dom}(V(t, \cdot)), \exists \bar{u} \in U(t)$ such that

$$
D_{\downarrow} V(t, x)(1, f(t, x, \bar{u})) \geq-L(t, x, \bar{u}) ;
$$

- for a.e. $t>0, \forall x \in \operatorname{int}(K) \cap \operatorname{dom}(V(t, \cdot)), \forall u \in U(t)$ the following two inequalities are satisfied

$$
D_{\downarrow} V(t, x)(-1,-f(t, x, u)) \geq L(t, x, u)
$$

and

$$
D_{\uparrow} V(t, x)(1, f(t, x, u)) \leq-L(t, x, u) .
$$

The above proposition follows easily from the dynamic programming principle and [27, Theorems 2.9, 4.2 and Corollary 2.7]. The equivalence results below allow to state the Hamilton-Jacobi inequalities involving sub and superdifferentials in terms of directional derivatives. Such equivalence is important, because it allows to deduce dynamic programming properties of a generalized solution which, in turn, yield uniqueness of solutions to (HJ) with the terminal condition (24). 
Proposition 9.5. Let $W: \mathbb{R}_{+} \times K \rightarrow \mathbb{R}$ be locally Lipschitz. Then

(1) the following two statements are equivalent:

(a) for a.e. $t>0, \forall x \in K, \exists \bar{u} \in U(t)$ such that

$$
D_{\downarrow} W(t, x)(1, f(t, x, \bar{u})) \geq-L(t, x, \bar{u}) ;
$$

(b) $p_{t}+H\left(t, x, p_{x}\right) \geq 0$ for a.e. $t>0, \forall x \in K$ and every $\left(p_{t}, p_{x}\right) \in$ $\partial_{+} W(t, x)$.

(2) the following two statements are equivalent:

$(a)^{\prime}$ for a.e. $t>0, \forall x \in \operatorname{int}(K), \forall u \in U(t)$,

$$
D_{\downarrow} W(t, x)(-1,-f(t, x, u)) \geq L(t, x, u) ;
$$

$(b)^{\prime} p_{t}+H\left(t, x, p_{x}\right) \leq 0$ for a.e. $t>0, \forall x \in \operatorname{int}(K)$ and every $\left(p_{t}, p_{x}\right) \in$ $\partial_{+} W(t, x)$.

Proof. Note that $(a) \Rightarrow(b)$ and $(a)^{\prime} \Rightarrow(b)^{\prime}$ by the very definition of superdifferential. From [27, Corollary 2.7 and Theorem 2.9] and [28, Corollary 3.2] we deduce that $(b) \Rightarrow(a)$ and $(b)^{\prime} \Rightarrow(a)^{\prime}$.

An analogous proposition can be stated also for the epigraph of $W$, we shall not dwell on it however.

Lemma 9.6. Let $W: \mathbb{R}_{+} \times K \rightarrow \mathbb{R}$ be locally Lipschitz and satisfying $(a)^{\prime}$ of Proposition 9.5. Then for all $0<\tau_{0}<\tau_{1}$ and any trajectory-control pair $(x(\cdot), u(\cdot))$ of $(5)$ on $\left[\tau_{0}, \tau_{1}\right]$, with $x\left(\left[\tau_{0}, \tau_{1}\right]\right) \subset \operatorname{int}(K)$, the function $w(\cdot)$ defined by

$$
w(t):=W\left(\tau_{1}, x\left(\tau_{1}\right)\right)+\int_{t}^{\tau_{1}} L(s, x(s), u(s)) d s
$$

satisfies $(x(t), w(t)) \in$ hyp $W(t, \cdot)$ for all $t \in\left[\tau_{0}, \tau_{1}\right]$.

Consequently, $W\left(\tau_{0}, x\left(\tau_{0}\right)\right) \geq W\left(\tau_{1}, x\left(\tau_{1}\right)\right)+\int_{\tau_{0}}^{\tau_{1}} L(t, x(t), u(t)) d t$.

Proof. Define $\phi(t)=W(t, x(t))$. By the Lipschitz continuity of $W$ for a.e. $t \in\left[\tau_{0}, \tau_{1}\right]$

$$
-\phi^{\prime}(t)=D_{\downarrow} W(t, x(t))(-1,-f(t, x(t), u(t))) \geq L(t, x(t), u(t)) .
$$

Integrating on $\left[t, \tau_{1}\right]$ implies

$$
\phi(t)-W\left(\tau_{1}, x\left(\tau_{1}\right)\right) \geq \int_{t}^{\tau_{1}} L(s, x(s), u(s)) d s=w(t)-W\left(\tau_{1}, x\left(\tau_{1}\right)\right) .
$$

Hence $W(t, x(t)) \geq w(t)$.

Similarly, we have the following lemma (after stating an analogue of Proposition 9.5 for the lower directional derivatives).

Lemma 9.7. Let $W: \mathbb{R}_{+} \times K \rightarrow \mathbb{R}$ be locally Lipschitz. If for a.e. $t>0$ and all $x \in \operatorname{int}(K)$,

$$
p_{t}+H\left(t, x, p_{x}\right) \leqslant 0 \quad \forall\left(p_{t}, p_{x}\right) \in \partial_{-} W(t, x),
$$


then for all $0<\tau_{0}<\tau_{1}$ and any trajectory-control pair $(x(\cdot), u(\cdot))$ of (5) on $\left[\tau_{0}, \tau_{1}\right]$ with $x\left(\left[\tau_{0}, \tau_{1}\right]\right) \subset \operatorname{int}(K)$, the function $w(\cdot)$ defined by

$$
w(t):=W\left(\tau_{0}, x\left(\tau_{0}\right)\right)-\int_{\tau_{0}}^{t} L(s, x(s), u(s)) d s
$$

satisfies $(x(t), w(t)) \in$ epi $W(t, \cdot)$ for all $t \in\left[\tau_{0}, \tau_{1}\right]$.

Consequently, $W\left(\tau_{0}, x\left(\tau_{0}\right)\right) \geq W\left(\tau_{1}, x\left(\tau_{1}\right)\right)+\int_{\tau_{0}}^{\tau_{1}} L(t, x(t), u(t)) d t$.

Proof. (of Theorem 9.1). To show that the locally Lipschitz value function satisfies (25) and (26) it is sufficient to apply Proposition 9.4 and definitions of super and subdifferentials. Let $\left(t_{0}, x_{0}\right) \in \mathbb{R}_{+} \times K$. To show that $W\left(t_{0}, x_{0}\right) \geq V\left(t_{0}, x_{0}\right)$, it is enough to consider the case when $V\left(t_{0}, x_{0}\right)>$ $-\infty$. Let $(\bar{x}(\cdot), \bar{u}(\cdot))$ be optimal at $\left(t_{0}, x_{0}\right)$. We apply $(\mathrm{NFT})$ theorem from [10] under either the assumption $\left(O P C^{\infty}\right)$ or $\left(I P C^{\infty}\right)$ and associate with any $\varepsilon>0$, the time interval $\left[t_{\varepsilon}, T_{\varepsilon}\right] \subset \mathbb{R}_{+}$and a trajectory-control pair $\left(x_{\varepsilon}(\cdot), u_{\varepsilon}(\cdot)\right)$ defined on $\left[t_{\varepsilon}, T_{\varepsilon}\right]$ satisfying $x_{\varepsilon}\left(\left[t_{\varepsilon}, T_{\varepsilon}\right]\right) \subset \operatorname{int}(K), \lim _{\varepsilon \rightarrow 0+} T_{\varepsilon}=$ $\infty, \lim _{\varepsilon \rightarrow 0+}\left(t_{\varepsilon}, x_{\varepsilon}\left(t_{\varepsilon}\right)\right)=\left(t_{0}, x_{0}\right)$ and

$$
\left|\int_{t_{\varepsilon}}^{T_{\varepsilon}} L\left(t, x_{\varepsilon}(t), u_{\varepsilon}(t)\right) d t-\int_{t_{\varepsilon}}^{T_{\varepsilon}} L(t, \bar{x}(t), \bar{u}(t)) d t\right| \leq \varepsilon .
$$

Then Lemmas 9.6 and 9.7 yield

$$
\begin{aligned}
W\left(\tau_{\varepsilon}, x_{\varepsilon}\left(\tau_{\varepsilon}\right)\right) & \geq W\left(T_{\varepsilon}, x_{\varepsilon}\left(T_{\varepsilon}\right)\right)+\int_{\tau_{\varepsilon}}^{T_{\varepsilon}} L\left(t, x_{\varepsilon}(t), u_{\varepsilon}(t)\right) d t \\
& \geq W\left(T_{\varepsilon}, x_{\varepsilon}\left(T_{\varepsilon}\right)\right)+\int_{t_{\varepsilon}}^{T_{\varepsilon}} L(t, \bar{x}(t), \bar{u}(t)) d t-\varepsilon .
\end{aligned}
$$

Hence, whenever $T_{\varepsilon}>S$ we get

$$
W\left(\tau_{\varepsilon}, x_{\varepsilon}\left(\tau_{\varepsilon}\right)\right) \geq W\left(T_{\varepsilon}, x_{\varepsilon}\left(T_{\varepsilon}\right)\right)+\int_{t_{\varepsilon}}^{\infty} L(t, \bar{x}(t), \bar{u}(t)) d t-\int_{T_{\varepsilon}}^{\infty} \alpha(t) d t-\varepsilon .
$$

Taking the limit when $\varepsilon \rightarrow 0+$, using (24) and the integrability of $\alpha$ on $\mathbb{R}_{+}$ we obtain $W\left(t_{0}, x_{0}\right) \geq V\left(t_{0}, x_{0}\right)$. Hence $W \geq V$.

Finally, Proposition $9.5(a)$ and a measurable viability theorem [28, Corollary 3.2] imply that for every $\left(t_{0}, x_{0}\right) \in \mathbb{R}_{+} \times K$ and $T>t_{0}$ there exists a trajectory-control pair $(\bar{x}(\cdot), \bar{u}(\cdot))$ such that

$$
W\left(t_{0}, x_{0}\right)-\int_{t_{0}}^{T} L(t, \bar{x}(t), \bar{u}(t)) d t \leq W(T, \bar{x}(T)) .
$$

Hence for any $T>S, W\left(t_{0}, x_{0}\right) \leq W(T, \bar{x}(T))+V\left(t_{0}, x_{0}\right)+\int_{T}^{\infty} \alpha(t) d t$. This, (24) and the integrability of $\alpha$ on $[S, \infty)$ imply that for every $\varepsilon>0$ we have $W\left(t_{0}, x_{0}\right) \leq V\left(t_{0}, x_{0}\right)+\varepsilon$. Consequently $W \leq V$ and we deduce that $(25)$ yields $W=V$. Similarly, (26) implies $W=V$.

\section{REFERENCES}

[1] Aseev, S.M.: On some properties of the adjoint variable in the relations of the Pontryagin maximum principle for optimal economic growth problems. Tr. Inst. Mat. Mekh. 19, 15-24, (2013) 
[2] Aseev, S.M., Kryazhimskiy, A.V.: The Pontryagin maximum principle and transversality conditions for a class of optimal control problems with infinite time horizons. SIAM J. Control Optim. 43, 1094-1119, (2004)

[3] Aseev, S.M., Veliov, V.M.: Maximum principle for infinite-horizon optimal control problems with dominating discount. Dynamics of Continuous, Discrete and Impulsive Systems, Series B: Applications \& Algorithms 19, 43-63, (2012)

[4] Aseev, S.M., Veliov, V.M., Maximum principle for infinite-horizon optimal control problems under weak regularity assumptions. In: Proceedings of the Steklov Institute of Mathematics 291, S22-S39, (2015)

[5] Aseev, S.M., Veliov, V.M.: Another view of the maximum principle for infinitehorizon optimal control problems in economics. Technical Report, IIASA, (2017)

[6] Aseev, S.M., Krastanov, M., Veliov, V.M.: Optimality conditions for discrete-time optimal control on infinite horizon. Pure Appl. Funct. Anal. 2, 395-409, (2017)

[7] Aubin, J.-P., Clarke, F.: Shadow prices and duality for a class of optimal control problems. SIAM J. Control Optim. 17, 567-586, (1979)

[8] Aubin, J.-P., Frankowska, H.: Set-Valued Analysis. Birkhäuser, Boston, Basel, Berlin, (1990)

[9] Basco, V., Cannarsa, P., Frankowska, H.: Necessary conditions for infinite horizon optimal control problems with state constraints. Mathematical Control and Related Fields (MCRF) 8, 535-555, (2018)

[10] Basco, V., Frankowska, H.: Lipschitz continuity of the value function for the infinite horizon optimal control problem under state constraints. In "Trends in Control Theory and Partial Differential Equations", Eds. Alabau, F., and al., Springer INdAM Series 32, (2019)

[11] Basco, V., Frankowska, H.: Hamilton-Jacobi-Bellman equations with timemeasurable data and infinite horizon. Nonlinear Differ. Equ. Appl. 26, https://doi.org/10.1007/s00030-019-0553-y (2019)

[12] Bettiol, P., Frankowska, H., Vinter, R.: Improved sensitivity relations in state constrained optimal control. Appl. Math. Optim. 71, 353-377, (2015)

[13] Benveniste, L.M., Scheinkman, J.A.: Duality theory for dynamic optimization models of economics: The continuous time case. J. Econom. Theory 27, 1-19, (1982)

[14] Cannarsa, P., Frankowska, H.: Value function, relaxation, and transversality conditions in infinite horizon optimal control. J. Math. Anal. Appl. 457, 1188-1217, (2018)

[15] Cannarsa, P., Sinestrari, C.: Semiconcave functions, Hamilton-Jacobi equations, and optimal control. Birkhäuser, Boston, MA, (2004)

[16] Cannarsa, P., Frankowska, H., Scarinci T.: Second-order sensitivity relations and regularity of the value function for Mayer's problem in optimal control. SIAM Journal on Control and Optimization 53, 3642-3672, (2015)

[17] Carlson, D.A., Haurie, A.: Infinite Horizon Optimal Control. Theory and Applications. Lecture Notes in Economics and Mathematical Systems, Springer-Verlag, Berlin, Heidelberg, (1987)

[18] Cernea, A., Frankowska, H.: A connection between the maximum principle and dynamic programming for constrained control problems. SIAM J. Control Optim. 44, 673-703, (2006)

[19] Clarke, F.H.: Optimization and Nonsmooth Analysis. Wiley, New York, (1983)

[20] Crandall, M.G., Lions, P.-L.: Viscosity solutions of Hamilton-Jacobi equations. Trans. Amer. Math. Soc. 277, 1-42, (1983)

[21] Crandall, M.G., Evans, L.C., Lions, P.-L.: Some properties of viscosity solutions of Hamilton-Jacobi equation. Trans. Amer. Math. Soc. 282, 487-502, (1984)

[22] Frankowska, H.: Optimal trajectories associated to a solution of contingent HamiltonJacobi equations. Appl. Math. Optim. 19, 291-311, (1989) 
[23] Frankowska, H.: Lower semicontinuous solutions of Hamilton-Jacobi-Bellman equation. SIAM J. Control Optim. 31, 257-272, (1993)

[24] Frankowska, H.: A priori estimates for operational differential inclusions. J. Diff. Eqs. 84, 100-128, (1990)

[25] Frankowska, H., Mazzola, M.: Discontinuous solutions of Hamilton-Jacobi-Bellman equation under state constraints. Calc. Var. and PDEs 46, 725-747, (2013)

[26] Frankowska, H., Mazzola, M.: On relations of the co-state to the value function for optimal control problems with state constraints. Nonlinear Differ. Equ. Appl. 20, 361-383, (2013)

[27] Frankowska, H., Plaskacz, S., Rzeżuchowski, T.: Measurable viability theorems and Hamilton-Jacobi-Bellman equations. J. Diff. Eqs. 116, 265-305, (1995)

[28] Frankowska, H., Plaskacz, S.: A measurable upper semicontinuous viability theorem for tubes. J. of Nonlinear Analysis, TMA 26, 565-582, (1996)

[29] Frankowska, H., Plaskacz, S.: Hamilton-Jacobi equations for infinite horizon control problems with state constraints. In: Proceedings of International Conference "Calculus of Variations and related topics", Haifa, 1998, Chapman and Hall/CRC Research Notes in Mathematics Series 411, 97-116, (2000)

[30] Frankowska, H., Plaskacz, S.: Semicontinuous solutions of Hamilton-Jacobi-Bellman equations with degenerate state constraints. J. Math. Anal. Appl. 251, 818-838, (2000)

[31] Frankowska, H., Vinter, R.B.: Existence of neighbouring feasible trajectories: applications to dynamic programming for state constrained optimal control problems. J. Optim. Theory Appl. 104, 21-40, (2000)

[32] Halkin, H.: Necessary conditions for optimal control problems with infinite horizons. Econometrica 42, 267-272, (1974)

[33] Khlopin, D.V.: Necessity of vanishing shadow price in infinite horizon control problems. J. Dyn. Con. Sys. 19, 519-552, (2013)

[34] Lykina, V., Pickenhain, S.: Weighted functional spaces approach in infinite horizon optimal control problems: A systematic analysis of hidden opportunities and advantages. J. Math. Anal. Appl. 454, 195-218, (2017)

[35] Michel, P.: On the transversality condition in infinite horizon optimal problems. Econometrica 50, 975-984, (1982)

[36] Pickenhain, S.: On adequate transversality conditions for infinite horizon optimal control problems - a famous example of Halkin. In : Dynamic Systems, Economic Growth, and the Environment, Springer, Berlin, 3-12, (2010)

[37] Pickenhain, S.: Infinite horizon optimal control problems in the light of convex analysis in Hilbert spaces. Set-Valued Var. Anal. 23, 169-189, (2015)

[38] Pickenhain, S.: Infinite horizon problems in the calculus of variations. Set-Valued Var. Anal. 2, 331-354, (2019)

[39] Ramsey, F.P.: A mathematical theory of saving. Economic Journal 38, 543-559, (1928)

[40] Sagara, N.: Value functions and transversality conditions for infinite-horizon optimal control problems. Set-Valued Var. Anal. 18, 1-28, (2010)

[41] Soner, H.M.: Optimal control with state-space constraints. SIAM J. Control Optim. 24, 552-561, (1986)

[42] Seierstad, A., Sydsaeter, K.: Optimal Control Theory with Economic Applications. North-Holland Publishing Co., Amsterdam, (1987)

[43] Seierstad, A., Sydsaeter, K.: Conditions implying the vanishing of the Hamiltonian at infinity in optimal control problems. Optim Lett. 3, 507-512, (2009)

[44] Tauchnitz, N.: The Pontryagin maximum principle for nonlinear optimal control problems with infinite horizon. J. Optim. Theory Appl. 167, 27-48, (2015)

[45] Vinter, R.B.: Optimal Control, Birkhäuser, Boston, (2000) 
[46] Von Weizsacker, C.C.: Existence of optimal programs of accumulation for an infinite time horizon. Review of Economic Studies 32, 85-92, (1965)

[47] Ye, J.: Nonsmooth maximum principle for infinite-horizon problems. J. Optim. Theory Appl. 76, 485-500, (1993)

CNRS, Institut de Mathématiques de Jussieu - Paris Rive Gauche, Sorbonne Université, Case 247, 4 Place Jussieu, 75252 Paris, France

E-mail address: helene.frankowska@imj-prg.fr 